\title{
Measurement Campaign on 5G Indoor millimeter Wave and Visible Light Communications Multi Component Carrier System
}

\author{
Kareem Ali ${ }^{1}$, John Cosmas ${ }^{1}$, Yue Zhang ${ }^{2}$, Hequn Zhang ${ }^{2}$, Ben Meunier ${ }^{1}$, Nawar Jawad ${ }^{1}$, Xun Zhang ${ }^{3}$, \\ Lina Shi ${ }^{3}$, James Gbadamosi ${ }^{4}$, Atanas Savov ${ }^{4}$
}

\begin{abstract}
This paper presents the technical performance results of a measurement campaign from a $5 \mathrm{G}$ indoor millimeter Wave (mmWave) and Visible Light Communications (VLC) multi component carrier system, which was developed in a Horizon 2020 research project called Internet of Radio-Light (IoRL). The measurement campaign was performed in the famous Integer House laboratory at the Innovation Park in Building Research Establishment in Watford, UK, which represents a typical European home environment. It includes four field test results: 1) VLC received signal quality measured as Error Vector Magnitude (EVM) against coverage, 2) mmWave received signal quality measured as EVM against coverage, 3) VLC location accuracy against a prescribed grid using received signal strength, 4) Comparison of measured and simulated Electromagnetic Field (EMF) strength against coverage. This measurement campaign not only tests the system concept in a realistic indoor home environment but also provides analysis of the results with practical recommendations on further technical enhancements required to improve the system performance and insights into viable commercial solutions and applications. Other environments in which this technology could be deployed were envisaged as: underground train platforms and tunnels, museums and supermarkets.
\end{abstract}

Index Terms - 5G indoor millimeter Wave and Visible Light Communications, 5G multicomponent carrier system, Visible Light Communication indoor location accuracy, simulated and measured Electromagnetic Field strength against coverage.

\section{INTRODUCTION}

$\mathrm{T}$ HE high demand in the usage of wireless communications in buildings is causing interference and congestion, whereas modern building materials restrict the propagation of Radio Frequency (RF) waves. Thus, there has been an interest in deploying cellular home networks (HeNBs) by the building owners as they operate in licensed spectrum, meaning they can avoid congestion and interference. However, the deployment of the HeNBs requires the approval of Mobile Network Operators (MNO) as a result of possible interference to the outside transmitted signal of the leading mobile network (eNB). Thus, every building that requires the deployment of a HeNB needs its own MNO to provide the approval for it, which makes it very inconvenient and costly for building owners.

\footnotetext{
${ }^{1}$ Brunel University, Kingston Ln, Uxbridge, UK

${ }^{2}$ University of Leicester, University Road, Leicester, UK

3 Institut supérieur d'électronique de Paris, rue de Vanves, Issy-lesMoulineaux, Paris, Fr

${ }^{4}$ Building Research Establishment, Bucknalls Lane, Watford, UK
}

Since the Internet of Radio Light (IoRL) project operates in an unlicensed $60 \mathrm{GHz}$ mmWave and visible light spectrum, it offers a solution for providing $5 \mathrm{G} \mathrm{HgNB}$ (Home gNB) broadband without the approval of MNOs, since the propagation characteristics of EM waves using the mentioned spectrum do not interfere with the transmitted outside $5 \mathrm{G}$ signal. Moreover, offering widespread broadband coverage within buildings since it uses radio-light access points installed within the buildings' lighting system.

The IoRL project proposes a $60 \mathrm{GHz}$ system; however, this was not financially viable when considering the development of the multiple Remote Radio Heads (RRH) required to evaluate the proposed location estimation systems. Therefore, a $40 \mathrm{GHz}$ system was developed instead due to cheaper and more readily available electronics.

This proof of principle system was tested at the Innovation Park in the Building Research Establishment Watford, UK. The Innovation Park features full-scale demonstration buildings that have been developed by industry partners, which display innovative design, materials and technologies that combine to address the development challenges facing regions across the world. Technology demonstration, research, testing, training and dissemination are key activities which underpin the operation and development of the Innovation Park, thus making it the ideal place to demonstrate the IoRL system in a home scenario in its famous Integer House, which has been showcasing new home technology for over 20 years. All numerical results of the VLC and mmWave measurement campaign are available on Zenodo database [16] and data from the EM Exposure Simulation are also available on Zenodo database [17].

The rest of this paper is organised as follows: Section II presents the system architecture. Section III details the experimental setup for the technical tests. Section IV describes the experimental procedure of practically evaluating coverage and localisation performance. Section $\mathrm{V}$ provides the results of the field tests. In Section VI, the details of the mmWave EMF exposure test results are presented. Section VII provides analysis of results for VLC coverage and location accuracy, mmWave Coverage and EMF exposure. Section VIII provides conclusions.

\section{SYSTEM ARCHITECTURE}

The IoRL layered architecture consists of four layers namely: Service, Network Function Virtualisation (NFV), 
Software Defined Network (SDN) and Access layer, as shown in Figure 1.

The Service layer refers to various services carried out on the data cloud home data centre server (HDSC) such as streaming video services, including services on the multi-core Multiaccess Edge Computing (MEC) Cloud Server such as geolocation, multimedia services, databases, security services...etc. Furthermore, to achieve successful interaction with back-end services, front-end mobile applications are run on the User Equipment (UE) device(s), i.e. Smart Phones, Tablet PCs, Virtual Reality Headsets and HDTVs.

The NFV layer refers to deploying the network functions in the form of NFVs through Virtual Network Function (VNF) technologies. Presenting IoRL services in this format enables resource slicing, which offers higher resource utilisation by hosting end-user services, network services, and third-party services on the same hardware resources. OpenStack Virtual Infrastructure Manager (VIM) was utilised to enable the NFV layer.

The SDN Layer refers to the networking paradigm utilised in the IoRL. SDN layer entails SDN Forwarding Devices (FDs) that routes IP packets to/from their 5G Layer 2/3 Protocol Processors, SDN controller that is integrated within Neutron project of OpenStack and the SDN applications that offer the logical intelligence of the network. The Network Function Virtualization Orchestrator (NFVO) invokes various virtual functions required for an Intelligent Home IP Gateway such as Access \& Mobility Management, Deep Packet Inspection and Network Security Functions.

The Access Layer consists of up to 32 Remote Radio Light Head (RRLH) Controllers. Each RRLH Controller drives up to four VLC and mmWave RRLH pairs transmitting the same Transport Block Sub-Frame over a single designated coverage area, typically a room or floor area of a building. This structure provides Multiple Input Single Output (MISO) transmission on downlink paths and Single Input Multiple Output (SIMO) on uplink paths.

A single RRLH Controller can provision each room or floor area in a building with its group of RRLHs, and intra-building handover performed between these areas with the aid of VLC and mmWave location-sensing application that continuously records the positions of UE in the building.

A UE can either obtain direct access to the Internet, by only using $5 \mathrm{G}$ protocols on the Access Layer interface to the UE, to deliver IP packets to the Network Layer and thence to the Server Applications in the Service Layer or obtain access to the Mobile Network Operator's (MNO) Evolved Packet Core (EPC)/5G Core (5GC), by using $5 \mathrm{G}$ protocols on the Access Layer interfaces to both the UE and EPC/5GC, to deliver IP packets to the Network Layer and thence to the applications supported by the MNO. For example, this latter approach allows applications, such as Facebook, on a Smartphone to be accessed on both the outside Mobile Network and the Intelligent Home Network by exploiting handover between them. The VNFs on the NFV Layer identify the destination of
IP packets, and the SDN Controller directs these IP packets to their appropriate destination.

Therefore, our proposed solution enables the building owner to have connectivity to different operators to facilitate the use of different devices registered with different operators and exploit the license free spectrum for accessing the home network.

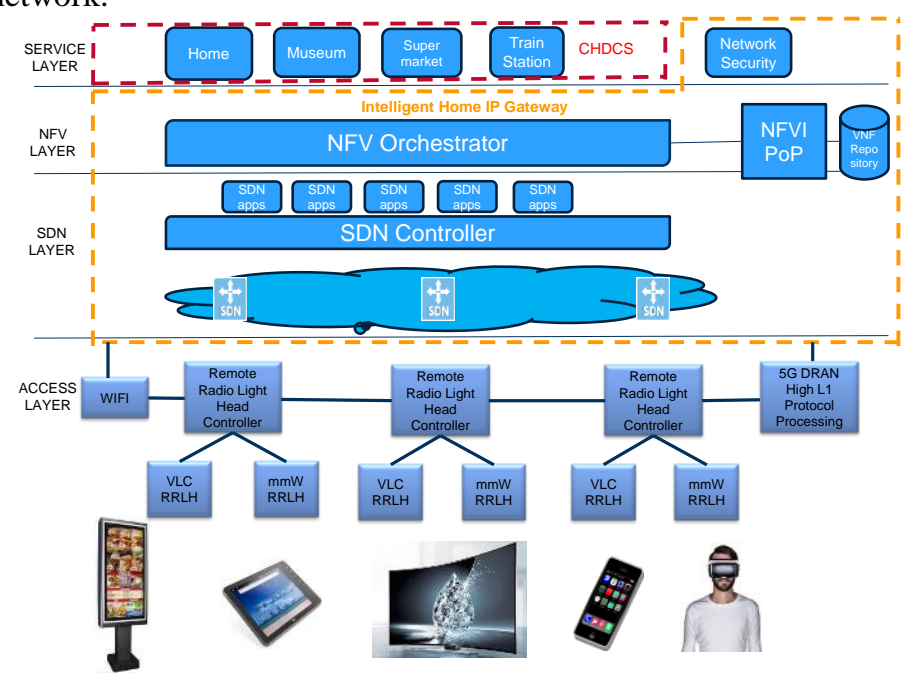

Figure 1: IoRL Layered Architecture

The Access Layer architecture employs a 10Gbps Common Public Radio Interface ring Ethernet to interconnect a Distributed Radio Access Network (DRAN) processor with up to 32 (RRLH) Controllers, each hosting two lower layer 1 processors. The first processor generates an IF signal to drive up to 4 VLC MISO modules with a 1 to 4 RF splitter and the second generates an IF signal to drive or be driven by up to 4 mmWave RF Duplex modules using a 1 to 4 RF splitter. The functional split between the RRLH Remote Unit and the DRAN in the Physical Layer is in-line with option 7 of the eCPRI architecture. The Upper PHY layer unit includes the interface with the MAC and upper RAN layers and mainly includes the FEC encoders (LDPC and Polar), decoders and drive through the 10Gbps Ethernet ring the data units along with their related control descriptors destined to the RRLH Controller units. The 10Gbps Ethernet ring can be looped between rooms in a building, connecting one RRLH to another, similar to the electric light circuit in a home. A 10 $\mathrm{MHz}$ Global Positioning Signal (GPS) reference clock is sent to the DRAN, RRLH Controller and UE for use in 5G synchronisation algorithms at these layers.

\section{EXPERIMENTAL SETUP}

The 5G multicomponent carrier indoor system contains six main components for both VLC and mmWave measurement campaign [1]. 1) A 5G base station including PHY Layer Central Unit, named DRAN (Distributed Radio Access Network) and RRLH Control Units. The DRAN carries out the tasks of the L1 upper layer, which mainly include the Forward Error Correction (FEC) encoding and decoding, beam management, distribution of the data to the RRLHs over the 10 Gbps Ethernet rings and the interface with the Medium Access 
Control (MAC) and higher layers. RRLH Control Units are responsible for the major part of the Physical (PHY) layer processing. Processing includes mainly the data modulation/ demodulation, precoding, Inverse Fast Fourier Transform (IFFT), air interface resource mapping, and antenna/Light Emitting Diode (LED) management. These units interface with the $10 \mathrm{Gbps}$ Ethernet ring and with the VLC and the mmWave modules via the $\mathrm{D} / \mathrm{A}$ and $\mathrm{A} / \mathrm{D}$, and through a switch and/or splitter/combiner. 2) A user equipment including a 5G New Radio (NR) baseband processing server hosts a signal analysing software and a Universal Software Radio Peripheral (USRP). This 5G NR baseband software can process the received signal from the USRP and perform the OFDM demodulation, channel estimation and zero-forcing equalisation to obtain the symbols. The USRP device connects to the 5G NR baseband processing server through $2 * 10$ Gigabit cables. The USRP device handles the input signal from the mmWave and VLC Receiver (RX) module. It converts the received $3.48 \mathrm{GHz}$ Intermediate Frequency (IF) signal to a baseband signal and delivers this to the data processing server. 3) mmWave Transmitter (TX) and RX modules generate and receive 40GHz Radio Frequency (RF) signals, respectively. 4) VLC Tx/Rx modules. 5) To make mmWave TX and RX modules work correctly, a Local Oscillator (LO) generator is used to provide a reference $13 \mathrm{GHz}$ signal. 6) A $10 \mathrm{MHz}$ reference signal is provided to the $13 \mathrm{GHz} \mathrm{LO}$ generator and the USRP device separately.

The system setup parameters, including the power levels, modulation schemes, bandwidth and carrier frequencies of these six central components are configured separately for the VLC and mmWave measurement campaign.

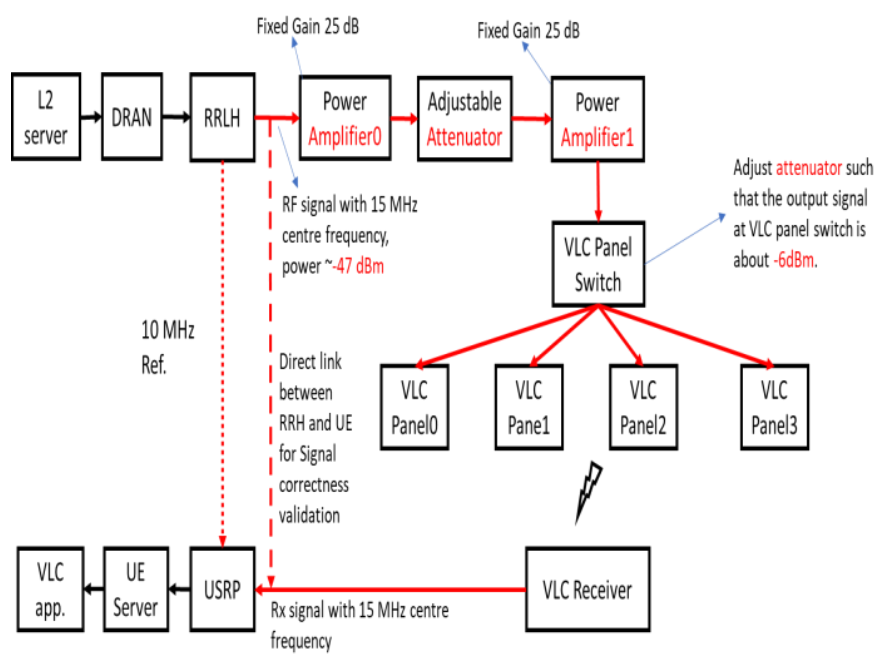

Figure 2: VLC Link

For the VLC link, the RRLH generates a signal with a 15 $\mathrm{MHz}$ centre IF frequency with a power of approximately -47 $\mathrm{dBm}$ from the $15 \mathrm{MHz}$ RF port. The signal is amplified to a proper power level and spit by the switch into 4 VLC panels. The red coloured line in the above diagram represents the RF cable with SubMiniature version A (SMA) connectors. The
VLC modulator inside each VLC panel is designed to accept a much higher input power. Generally, the higher the input power, the better the signal quality is for VLC modulation. However, a protective input power limit is implemented to prevent the VLC modulator from being damaged.

Consequently, the VLC panel input should be no more than $-6 \mathrm{dBm}$. In order to achieve good signal quality, two-stage amplifiers are required to provide a $-6 \mathrm{dBm}$ power level signal to the VLC panel. Additionally, to cope with uncertain cable attenuation, an adjustable attenuator is connected between the two amplifiers.

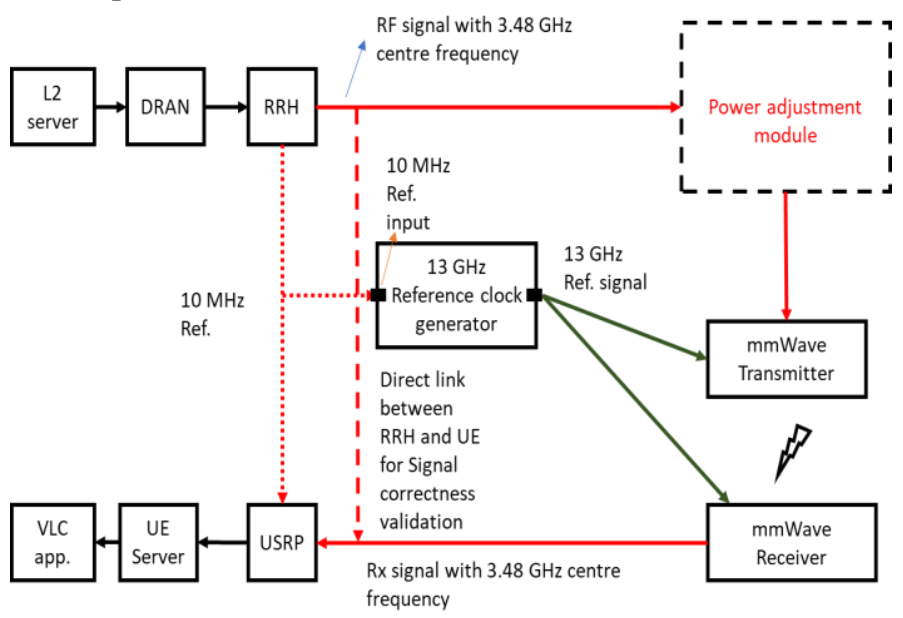

Figure 3: mmWave Link

As for the mmWave link, the RRLHs generate a signal with a $3.48 \mathrm{GHz}$ centre frequency from the $3.48 \mathrm{GHz} \mathrm{RF}$ port at $27 \mathrm{dBm}$ power. In addition to the signal path, the mmWave system requires a separate $13 \mathrm{GHz}$ reference signal, which was generated by a signal generator that was synchronised by the $10 \mathrm{MHz}$ reference. This is generated by the external frequency source, which is driven by the RRLH $10 \mathrm{MHz}$ reference signal. It is noted that the deep green coloured lines in this diagram represent a high-frequency cable which carry the $13 \mathrm{GHz}$ reference signal. The red coloured lines represent sub- $6 \mathrm{GHz}$ cables. The transmitted signal parameters for the mmWave and VLC link are listed in TABLE 1.

TABLE 1

Transmitted Signal Parameters

\begin{tabular}{|c|c|c|}
\hline Parameter & mmWave link & VLC Link \\
\hline $\begin{array}{c}\text { RF Carrier } \\
\text { frequency (Hz) }\end{array}$ & $60 \mathrm{GHz}$ or $40 \mathrm{GHz}$ & $\begin{array}{c}\text { Visible Light } \\
400-800 \mathrm{THz}\end{array}$ \\
\hline $\begin{array}{c}\text { IF Carrier } \\
\text { frequency (Hz) }\end{array}$ & $3.48 \mathrm{GHz}$ & $15 \mathrm{MHz}$ \\
\hline $\begin{array}{c}\text { Transmitted } \\
\text { power out of } \\
\text { RRLH (dBm) }\end{array}$ & -27 & -47 \\
\hline $\begin{array}{c}\text { Actual Bandwidth } \\
\text { (MHz) }\end{array}$ & 100 & 10 \\
\hline Modulation & $64 Q A M$ & QPSK \\
\hline RX USRP Gain (dB) & 0 & 0 \\
\hline Ch Cable loss (dB) & 3 & 3 \\
\hline
\end{tabular}


This article has been accepted for publication in a future issue of this journal, but has not been fully edited. Content may change prior to final publication. Citation information: DOI10.1109/TBC.2021.3120918, IEEE Transactions on Broadcasting

During the measurement campaign, the mmWave and VLC link transmitter parameters are fixed for all scenarios while the transmission distance, angle and location are varied.

The clock synchronisation system requires the RRLH to provide the $10 \mathrm{MHz}$ reference signal for both the UE and mmWave module. Figure 4: illustrates the $10 \mathrm{MHz}$ reference signal distribution.

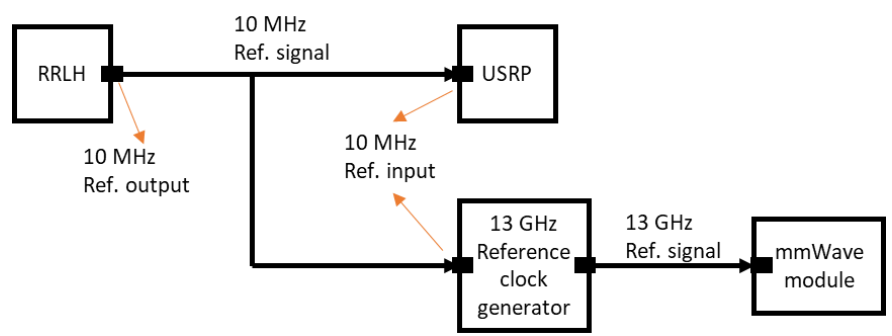

Figure 4: 10MHz Reference Distribution

\section{EXPERIMENTAL PROCEDURE}

This section describes the methodology used to practically evaluate both the coverage and localisation performance of the IoRL system within a home sitting room scenario. For coverage tests, a live feed readout program (MobaXterm) on the user terminal records the Error Vector magnitude (EVM) of the received signal at various points within the indoor environment. Due to various time and system restrictions, only the localisation through the VLC system is evaluated within this campaign. The localisation performance investigation involves collecting VLC Received Signal Strength (RSS) data using a custom Python code within the user terminal. To evaluate the VLC localisation performance, the RSS data is used to provide a position estimate. The resultant error is given by the Euclidean distance between the estimated and true position of the receiver. This study examines downlink transmission only such that the IoRL transmitters are fixed to the RRLHs, while the mobile receiver module requires manual re-positioning.

The home environment consists of laminate wooden flooring, a leather sofa and a pair of wooden chairs around the edge. An aluminium profile rig of dimensions $(2.5 \mathrm{~m} * 2.5 \mathrm{~m} * 2.2 \mathrm{~m})$ concerning $\mathrm{L} * \mathrm{~W} * \mathrm{H}$ provides a nonintrusive method to support and mount all required IoRL hardware during the measurement campaign. As shown in Figure 5, the experimental system setup has four square RRLHs mounted to the rig facing directly downwards onto a floor-level $\mathrm{cm}$ grid.

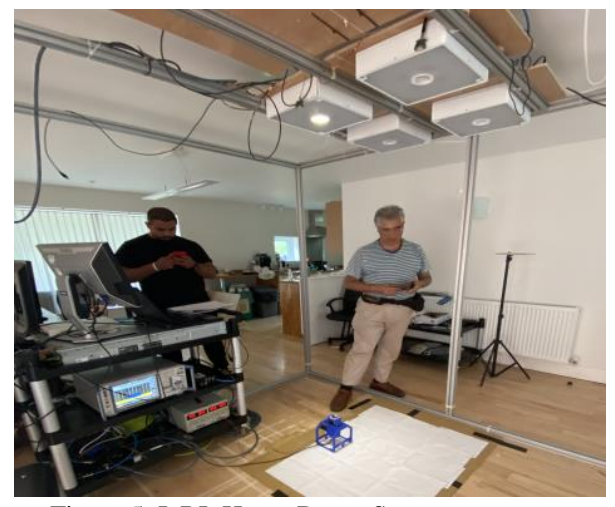

Figure 5: IoRL Home Demo Setup

\section{A. VLC coverage}

The VLC coverage is measured for each RRLH individually. Initially, using a plumb line, the centre point of the VLC light source is mapped onto a floor-level $\mathrm{cm}$ spaced grid. This centre point defines the respective RRLH origin. Then, the VLC receiver module is placed directly on the origin point, with the photodiode facing directly upwards, in a vertical orientation. EVM Measurements of the received signals are recorded at $6 \mathrm{~cm}$ spacings, up to $54 \mathrm{~cm}$, outward from the origin. This process is conducted at each 45-degree angle across the floor. EVM measurements are left to stabilise between positioning the receiver and performing the data recording. Notably, due to the long focal length of the lens used to focus the received light, a minor horizontal translation from the origin causes the received light path to 'miss' the receiver photodiode entirely, thus eliminating any received signal. Consequently, a custom gimbal houses the receiver for all VLC measurements. The gimbal maintains the receiver photodiode's centre point position along the 2D floor grid while providing a means to angle the receiver towards the respective light source, thereby maintaining Line of Sight (LOS). We repeated this process for each of the four RRLHs.

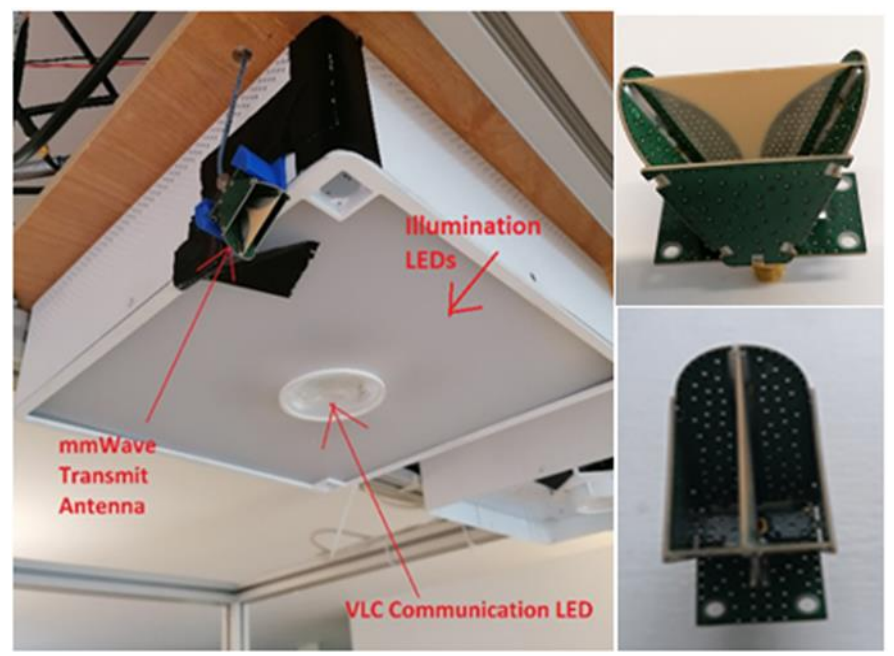

Figure 6: Remote Radio Light Head

\section{B. mmWave coverage}

This study measures the performance of a single mmWave polarised horn antenna. The antenna is mounted to the side of a RRLH, as shown in Figure 6 but orientated to emit directly downward. Taking the centre point of the horn antenna vertically down to floor level formed the grid's origin. The grids $\mathrm{x}$ and $\mathrm{y}$ axes are made about the horn antenna's orientation to maintain alignment between the receiver and transmitter. The $\mathrm{x}$-axis is parallel to the long edge of the horn antenna. To examine both a Non-LOS (NLOS) and LOS scenario, the receiver module is placed along the grid with a vertical and angled orientation, respectively, using the gimbal. An 'angled orientation' refers to using the receiver gimbal. EVM Measurements are left to stabilise between positioning the receiver and performing the data recording. These tests conducted both at floor level and a second tabletop height of 
$0.7 \mathrm{~m}$ from floor level. Additionally, the transmitting antenna is set to 30 degrees and 40 degrees from its vertical axis; however, it maintains the same origin point about the antennas base. Throughout these angled transmitter experiments, the receiver is used in both a vertical and angled orientation.

\section{VLC Location Experiments}

Similarly to the VLC coverage experiment, all 4 RRLH VLC sources' centre point positions are mapped vertically down to a $\mathrm{cm}$ grid at floor level and a tabletop height of $0.7 \mathrm{~m}$ using a plumb line. For calibration, initial records of the vertical attenuation of each VLC source are conducted by measuring the RSS directly below each RRLH at its mapped centre points at both floor and tabletop height. The measured data consists of a series of power reference signals to determine the signals RSS. At every designated position along the $2 \mathrm{D}$ grid, the receiver collects individual measurements sequentially from each RRLH. Due to occlusion, the receiver module must be angled towards each RRLH to establish LOS. The receiver gimbal ensures the centre point of the receiver is consistent but angled towards the correct RRLH.

\section{Estimated Measurement Errors}

A laser range tool, the Leica DISTO D2 with a $+/-1.5 \mathrm{~mm}$ tolerance, provides a reference for all mmWave grid layouts and vertical height measurements throughout the measurement campaign. Practical errors present themselves primarily in the positioning and angling of the receiver module and using a plumb line for centre point positioning. Manual positioning of the receiver using a crosshair provides at maximum an estimated $+/-1 \mathrm{~mm}$ tolerance, whereas angles are determined using a protractor with an estimated +/- 1-degree precision. Repeated plumb line measurements determine the precision to be an estimated +/- $1 \mathrm{~mm}$.

E. Human exposure to time-varying electric, magnetic and electromagnetic fields experiments

\section{1) Background}

EMF experimental procedure consisted of simulating the EMF exposure using simulation tools and then validating the simulations using field measurements within a restricted area to estimate the exposure throughout the room.

The International Commission on Non-Ionizing Radiation Protection (ICNIRP) offers scientific guidance and advice on the health and environmental effects of non-ionising radiation (NIR) to protect people and the environment from harmful NIR exposure. Non-ionising radiation refers to electromagnetic radiation such as infrared, ultraviolet, light and radio waves.

The electromagnetic spectrum comprising the frequency range from $100 \mathrm{kHz}$ to $300 \mathrm{GHz}$ is called High Frequency (HF), with mmWave corresponding to a range of frequencies between 30 to $300 \mathrm{GHz}$ (10mm to $1 \mathrm{~mm}$ wavelengths).

Various applications are emerging in this band, including imaging and monitoring systems and wireless telecommunications. The dangerous effect of HF exposure to human health and safety is the heating of exposed tissue. High- frequency fields can penetrate the body, causing the vibration of charged or polar molecules inside. So, the higher the frequency the lower the penetration depth.

\section{2) Computational Electromagnetics}

There are a lot of methods of calculation with varying degrees of complexity and accuracy. For the exposure assessment, it is advised to use the simplest appropriate method. The choice is highly dependent on the field region in which the investigation points are located in relation to the radiating source [15].

The required data put in order of the growing level of accuracy of the exposure assessment by the calculation are the following:

- Operating frequency;

- Distance to the transmitting antenna;

- Maximum equivalent isotropic radiated power (EIRP).

The next step in approving accuracy is obtaining radiation patterns of the transmitting antenna. Complete knowledge of the radiating sources is not entirely needed when taken measurements if the equipment covers the full range of frequencies and knowing at least the range of frequencies to be measured. However, suppose the measurements are made with wideband equipment (without frequency selection or shaped response). In that case, the results of such measurement will be conservative because it requires the use of the limit value, which is more restrictive. Nevertheless, in all measurements, the information concerning the radiating sources is beneficial and makes the measurements more accurate and reliable.

The following data are valuable during measurements (for each radiating source):

- operating frequency - this allows the use of a probe that has a band covering all operating frequencies;

- distance to the transmitting antenna - this allows one to determine the field region (for each operating frequency) and to choose a proper measurement procedure;

- maximum equivalent radiated power (ERP) - this allows estimation of the required dynamic range of the measurement equipment and the expected levels of the measured values;

- whether the antennas are operating at the maximum transmitter power at the time of the measurements;

- modulation characteristics - especially pulsed, intermittent or continuous operation.

Usually, this information can be obtained from the documentation of the transmitting systems. In addition, some data can be obtained during the site inspection (e.g., distances to the transmitting antennas, operating frequencies based on the types and sizes of the transmitting antennas) [15].

I. Measurements of electromagnetic fields

As part of the evaluation process that was planned, software modelling took place. This section explains the choice of software, preparing of the simulation model and yielded results.

3) Software selection and Modelling

There are two electromagnetic simulation software packages were considered, FEKO and WinProp from Altair. 
This article has been accepted for publication in a future issue of this journal, but has not been fully edited. Content may change prior to final publication. Citation information: DOI10.1109/TBC.2021.3120918, IEEE Transactions on Broadcasting

FEKO is a comprehensive computational electromagnetics (CEM) software that is widely used in the automobile, defence, aerospace and telecommunication industries. FEKO offers several frequency and time domain EM solvers, including Method of Moments (MoM) and Finite Difference Time Doman (FDTD). The hybridisation of these approaches allows efficient analysis of a broad spectrum of EM problems, including RF components and biomedical systems, microstrip circuits, the placement of antennas on electrically large structures antennas, and the calculation of scattering as well as the investigation of electromagnetic compatibility (EMC).

FEKO and WinProp are used globally across multiple industries, including aerospace, communications, automotive, defence, and consumer electronics, to reduce the time-tomarket. FEKO addresses the broadest set of high-frequency electromagnetics applications. It allows teams to optimise wireless connectivity, including 5G. Moreover, it ensures Electromagnetic Compatibility (EMC), scattering analysis and perform Radar Cross Section (RCS).

Due to the complex nature of near field, a software simulation is preferred over measurements. In the near field, the EM field structure could also be highly inhomogeneous, and there could also be substantial variations from the planewave impedance of $377 \mathrm{ohms}$. That may be where it would be almost pure electric (E) fields in some regions and almost pure magnetic $(\mathrm{H})$ fields in others. Exposures in the near field are more difficult to specify because both fields must be measured and because their field patterns are more complex.

Full-wave analysis techniques (e.g. methods requiring Maxwell's equations to be solved anywhere) are essentially used when high accuracy is desired for the evaluation of RF fields. For example, for RF field strength, power density or Specific Absorption Rate (SAR) evaluation in source region I (the reactive near-field of the antenna(s)) where ray tracing methods cannot be employed with sufficient accuracy. An accurate and realistic numerical model of the antenna shall be created for a complete wavefield analysis.

MoM or FDTD method is used to solve integral equation formulations of Maxwell's equations numerically. In principle, the radiated electromagnetic fields are obtained by following a two-step procedure.

a) First, structures that are represented with a mesh are replaced by equivalent currents. Then, a matrix is derived, which represents the effect of each element/segment on each other segment/element, and the surface currents are solved.

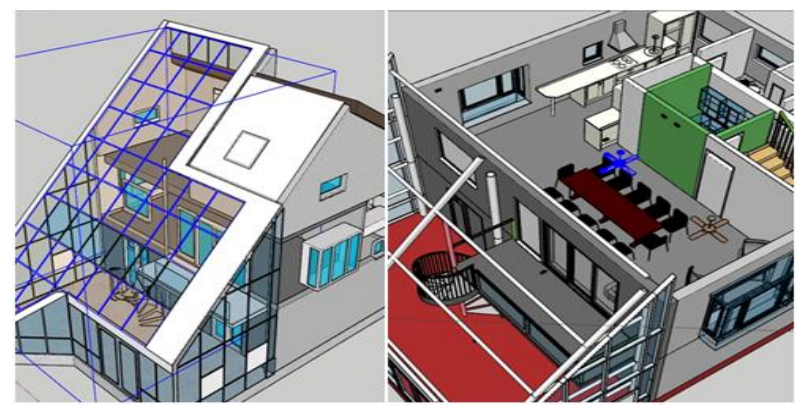

Figure 7: Detailed model of home scenario b) Secondly, these currents are integrated to obtain the electric and magnetic fields at the points of interest.

\section{4) Simulation Model preparation}

Two models were prepared for each of the chosen Altair's software product components: WinProp and FEKO. A model the size of a house would be very computationally expensive for FEKO, if the solver of choice were MoM. However, that is not the case for WinProp, which uses ray tracing as a method of solving. Therefore, the idea was to use a suitable approach to compute complex near field - method of moments, and ray tracing method for the far field region.

\section{RESULTS OF FIELD TESTS}

This section provides all the different field tests that was carried out and their results. mmWave and VLC were tested pointing vertical down to determine, which RHL provides the most coverage area. Both were angled towards the transmitter at 30 and 40 degrees to see if there are any improvements in the coverage area. In addition, the tests took place at two different heights at ground level and at $70 \mathrm{~cm}$ distance from the RHL. This was to see if there would be any improvements in their EVMs.

\section{A. VLC Coverage Results}

The photodiode receiver was not angled towards the communication LED with illumination LEDs off. As illustrated in Figure 8, the results show the coverage for 4 VLC LED TXs EVM test at $2 \mathrm{~m}$ distance from the transmitter and the $\mathrm{Rx}$ photodiode angle vertically up. RRLH A performed the best, providing a coverage area of radius $0.3 \mathrm{~m}$. A dramatic increase in EVM is observed when the coverage exceeds $0.3 \mathrm{~m} \times 0.3 \mathrm{~m}$.

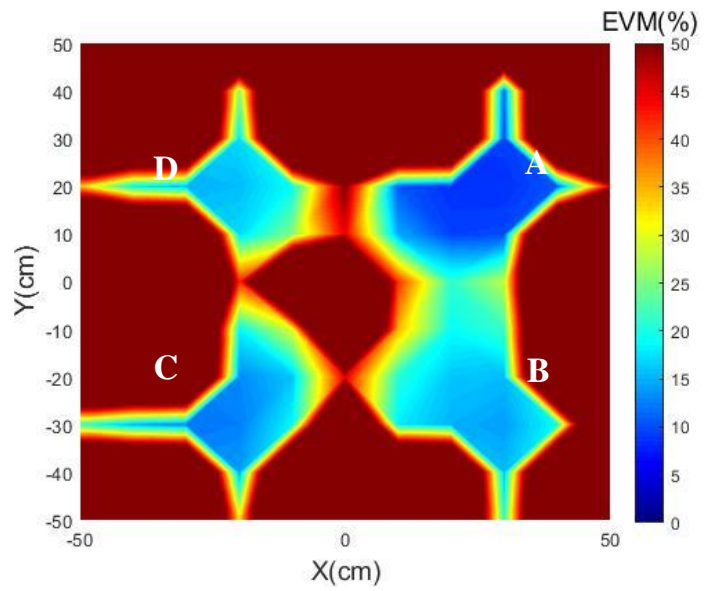

Figure 8: Four VLC TX LEDs pointing vertically down and Rx PD NonAngled (pointing vertically up), EVM Test results at ground level

The results of each of the 4 VLC LED TXs coverage is shown in Figure 9. The test was conducted at $2 \mathrm{~m}$ distance with illumination LEDs off and Rx Photodiode (PD) angled towards the communication LED. The best performing RLH is $\mathrm{A}$, as it provides a coverage area of a radius of $0.5 \mathrm{~m}$. 

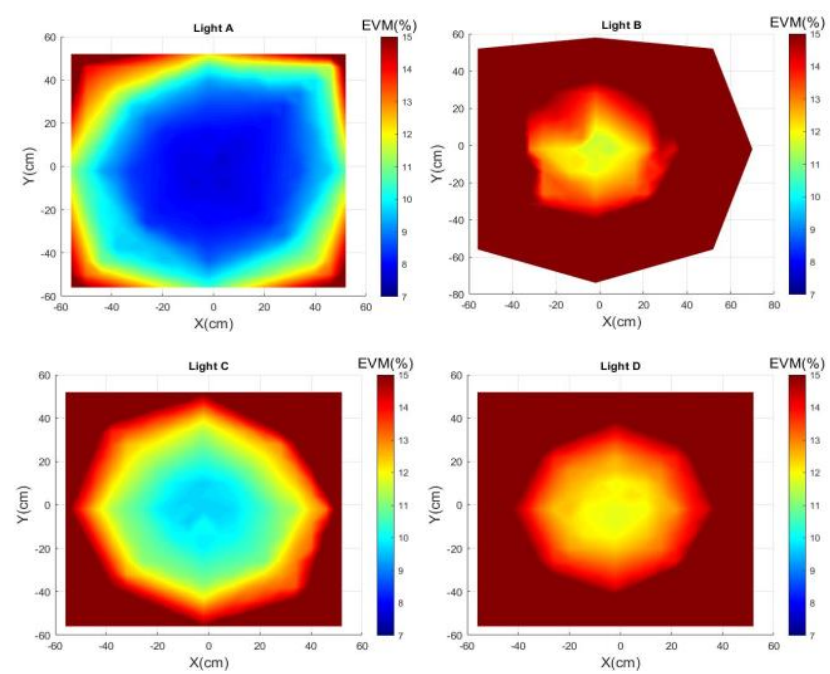

Figure 9: 4 Four VLC TX LEDs pointing vertically down and Rx PD Angled towards Tx, EVM Test results at ground level

\section{B. VLC Localisation Results}

A total of 27 test points, in which all four RRLHs provided coverage, are evaluated for VLC localisation performance. At each test point, on the $\mathrm{cm}$ grid, the RSS of each VLC source is measured by adjusting the receiver angle such that the receivers' PD establishes LOS with the respective VLC source. The resulting data is processed offline to provide an estimate of each position. The respective positioning error (PE) is determined by taking the Euclidean distance between the receivers true position during testing and the estimated location. The distribution of PE is shown in Figure 10, where the orange points represent the estimated points, and the blue points represent the test points, respectively. The minimum and maximum PE was $0.55 \mathrm{~cm}$ and $11.94 \mathrm{~cm}$, respectively, with an average of $5.28 \mathrm{~cm}$, as shown in Table 2 below. An enhanced RSS positioning algorithm [2], using an Artificial Intelligence (AI)-based algorithm, was applied to figure out the optimum position of four Remote Light Heads to reduce the location error of measurements taken in a grid of points within the coverage area. It estimates the positions the LEDs such that the best location measurement perfomance is obtained.Moreover, the cumulative distribution function (CDF) of the estimated points is plotted in Figure 11. It can be found that the current experimental testbed can reach a positioning accuracy of $10 \mathrm{~cm}$ with a confidence of $81.48 \%$.

TABLE 2

VLC Location Error

\begin{tabular}{|c|c|c|}
\hline Min PE $(\mathrm{cm})$ & Max PE $(\mathrm{cm})$ & Mean PE $(\mathrm{cm})$ \\
\hline 0.55 & 11.94 & 5.28 \\
\hline
\end{tabular}

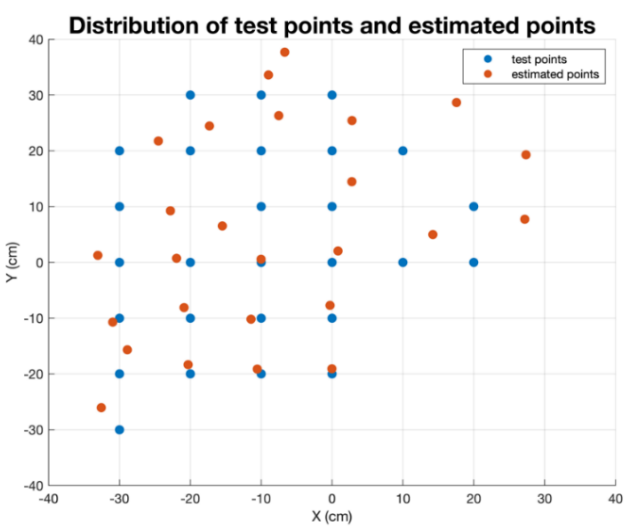

Figure 10: Distribution of test points and estimated points

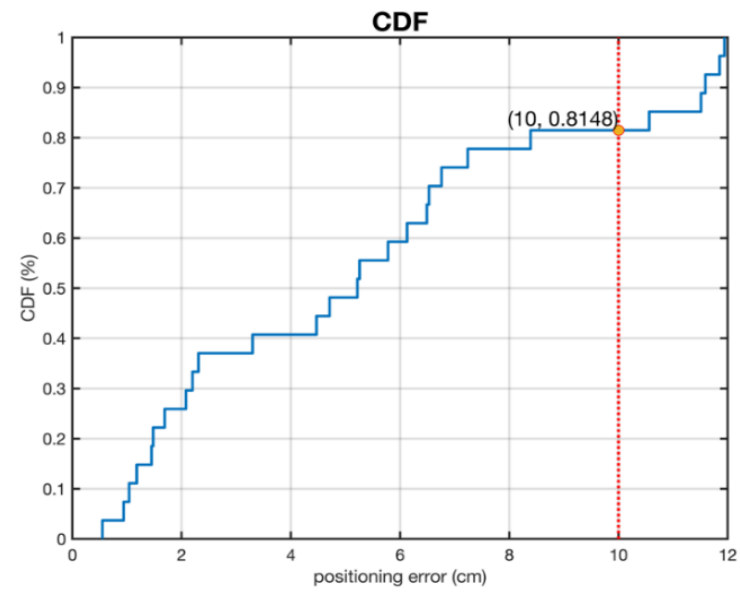

Figure 11: CDF of position error $(<10 \mathrm{CM})$

Figure 11, shows the cumulative distribution function (CDF) plot of position error $(<10 \mathrm{~cm})$, where $81.48 \%$ of the estimated position errors are less than $10 \mathrm{~cm}$.

\section{C. mmWave Downlink Coverage Results}

\section{1) mmWave Transmit Antenna Pointing Vertically Down}

Figure 12 illustrates the coverage results for a mmWave EVM test at a height $0.7 \mathrm{~m}$ above the ground $(1.3 \mathrm{~m}$ from the Tx antenna). This test is conducted with and without the $\mathrm{Rx}$ antenna angled towards the Tx antenna. The Tx is emitting vertically down.

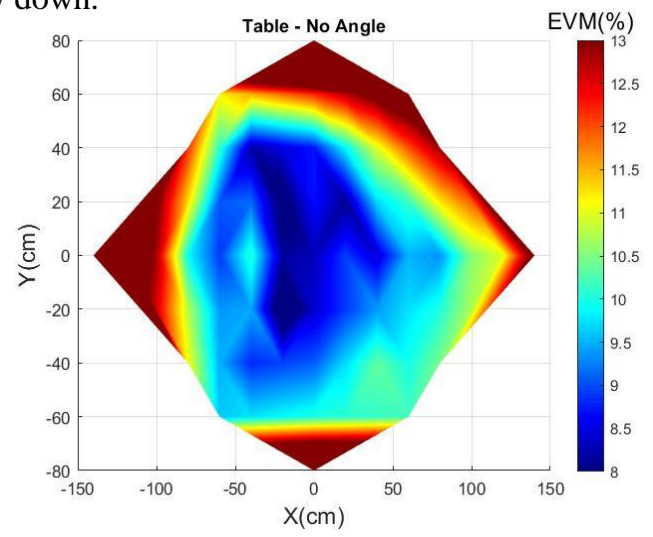

(a) Without angling Rx toward Tx antenna

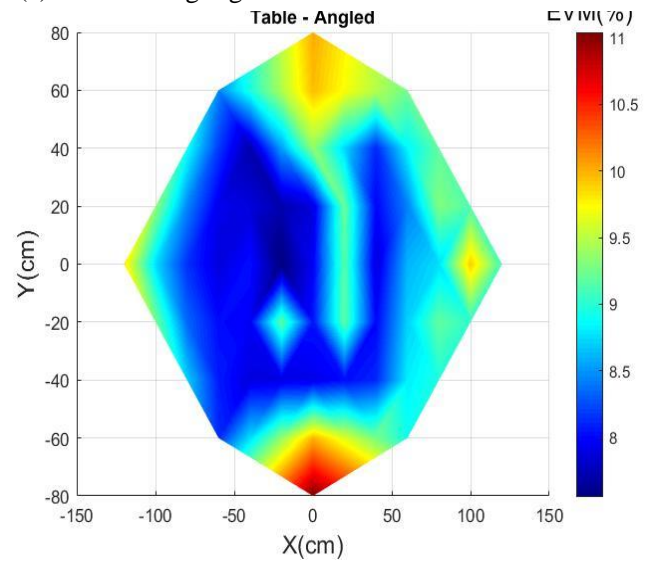

(b)With angling Rx toward Tx antenna

Figure 12: One mmWave TXs, receiver at $0.7 \mathrm{~m}$ above ground EVM Test

The EVM in most of the coverage region was $=<8 \%$ making it suited for 64-QAM transmission (for 4-QAM this is $12 \%$ 
and for 16-QAM this is $10 \%)$. The maximum propagation in the $\mathrm{x}$-axis $(1.2 \mathrm{~m})$ is more significant than in the $\mathrm{y}$-axis $(0.8 \mathrm{~m})$ due to the physical construction of the PCB Horn antenna where the horn slant is only applied in the $\mathrm{x}$-axis and not in the $y$-axis. Note: the antenna is polarised in one direction, so the transmit and receive antennas must be oriented in the same direction to maintain polarisation alignment with each other; otherwise, the reception will be poor.

The sample results in Figure 13 shows the coverage for one mmWave EVM test measurements at ground level $(2.1 \mathrm{~m}$ from Tx antenna). The test was conducted with and without the $\mathrm{Rx}$ antenna angled towards the Tx antenna. The Tx is emitting directly vertically down.

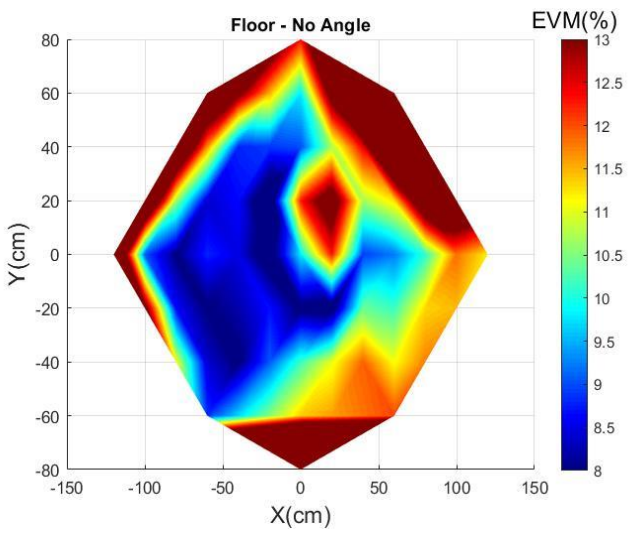

(a) Without angling Rx toward Tx antenna

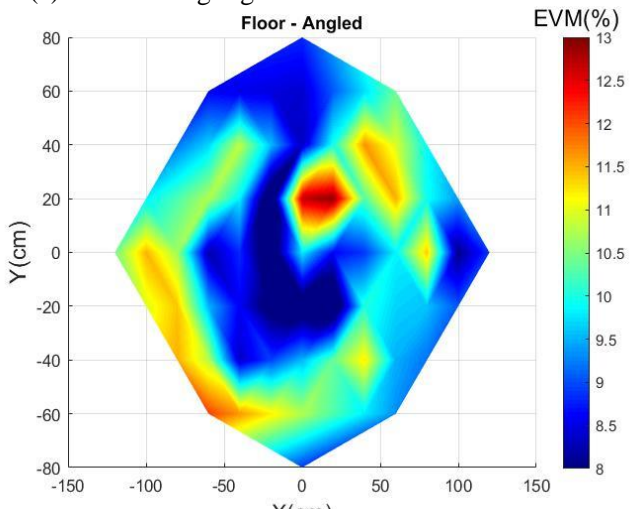

(b) With angling Rx toward Tx antenna

Figure 13: One mmWave TXs, receiver at $0 \mathrm{~m}$ ground level EVM Test

The EVM in most of the coverage region was $=<8 \%$ making it suited for 64-QAM transmission. The results show that angling the Rx towards the Tx antenna shows better EVM than non-angled. The performance was also observed to improve when the receiver is placed on the table at $0.7 \mathrm{~m}$. This was due to the shrouding effect of the table to reflections from the floor.

\section{2) mmWave Transmit Antenna Point $30^{\circ}$ from Vertical about antenna $y$-axis}

The transmit antenna is angled along the $\mathrm{x}$-axis by 30 degrees. The receiving antenna is angled towards the transmitting antenna to improve LOS resulting in a coverage area of at least $1.6 \mathrm{~m}$ in both axes, as shown in Figure b. In contrast, the signal quality degrades substantially when the receiver is not angled but directed directly upwards. As a result, the usable coverage is restricted to $0.8 \mathrm{~m}$ in the $\mathrm{x}$ - direction and $1.2 \mathrm{~m}$ in the y-direction. These measurements were taken on the 17th of September 2020.

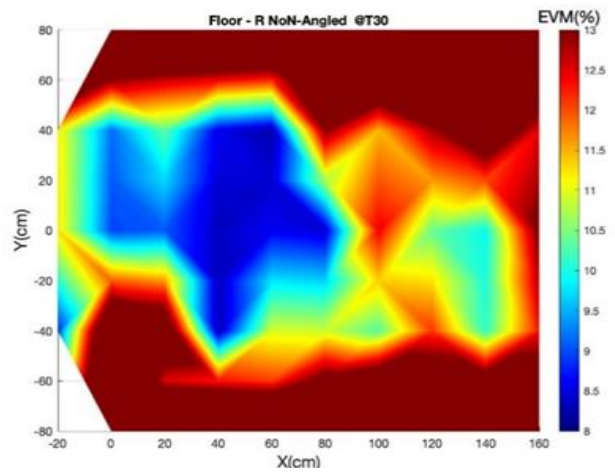

(a) Without angling Rx toward Tx antenna

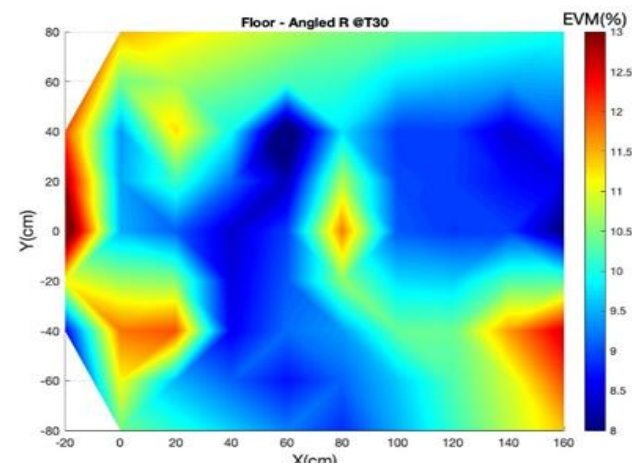

(b) With angling Rx toward Tx antenna

Figure 14: One mmWave TXs angled at $30^{\circ}$, receiver at $0 \mathrm{~m}$ above ground EVM Test

This test was repeated on the 22nd of September and results are shown in Figure. The new results were unexpectedly better. However, this indicates that the mmWave system's performance depends on external factors, which could not be identified, given that the experimental setup was unchanged.

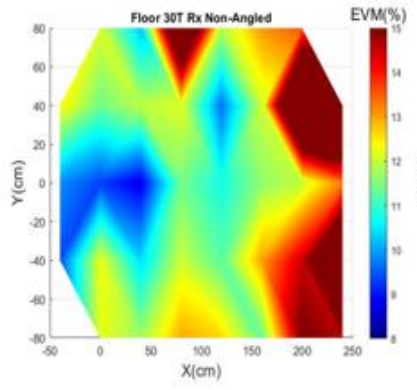

(a) Without angling $\mathrm{Rx}$ towards $\mathrm{Tx}$ antenna

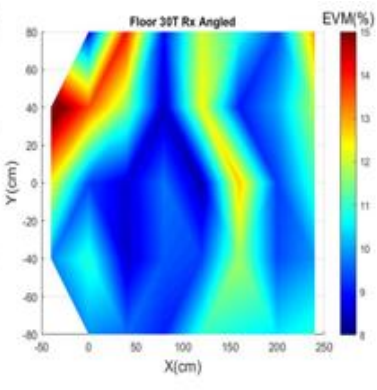

(b) With angling $\mathrm{Rx}$ towards $\mathrm{Tx}$ antenna
Figure 15: One mmWave TXs angled at 30, receiver at $0 \mathrm{~m}$ above ground EVM Test

Finally, The measurements were taken once more, where the transmitting antenna was angled at 30 degrees, but the $\mathrm{Rx}$ antenna is positioned at $0.7 \mathrm{~m}$ height above the ground. Results for both a vertical and angled Rx orientation are shown in Figu. 
This article has been accepted for publication in a future issue of this journal, but has not been fully edited. Content may change prior to final publication. Citation information: DOI10.1109/TBC.2021.3120918, IEEE Transactions on Broadcasting

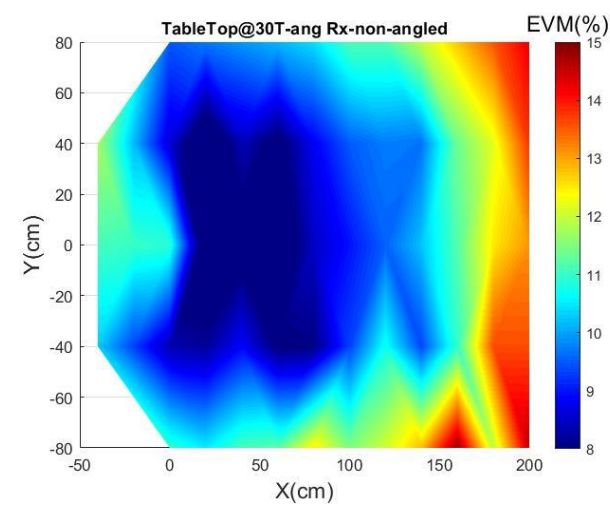

(a) Without angling Rx toward Tx antenna

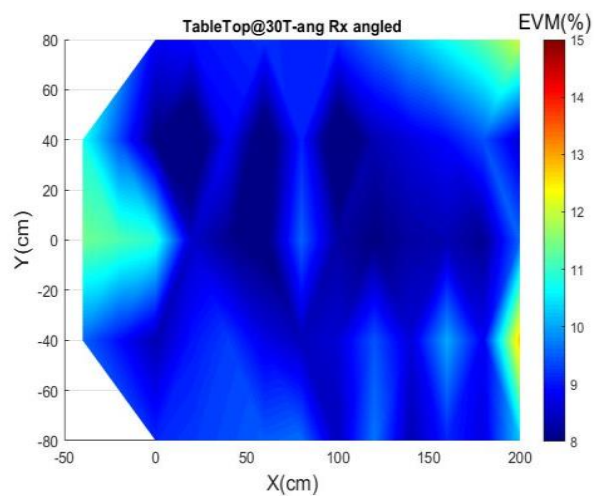

(b) With angling Rx toward Tx antenna

Figure 16: One mmWave TXs angled at $30^{\circ}$, receiver at $0.7 \mathrm{~m}$ above ground EVM Test

The performance of the mmWave system does improve with the Rx angled towards the Tx antenna, and it improves near the location of the Tx antenna.

\section{3) mmWave Transmit Antenna Point $40^{\circ}$ from Vertical about antenna y-axis}

The transmit antenna is angled along the $\mathrm{x}$-axis by 40 degrees. The receiving antenna was directed towards the transmitting antenna producing a coverage area of at least $1.6 \mathrm{~m}$ in both $\mathrm{x}$ and $\mathrm{y}$ direction as shown in Figure17.

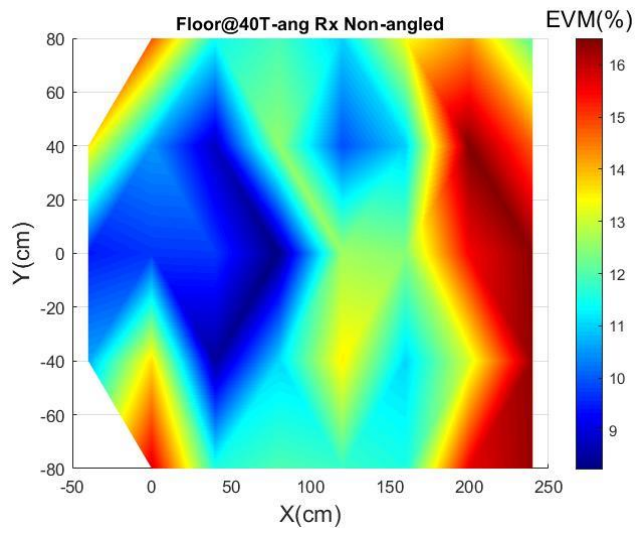

(a) Without angling Rx toward Tx antenna

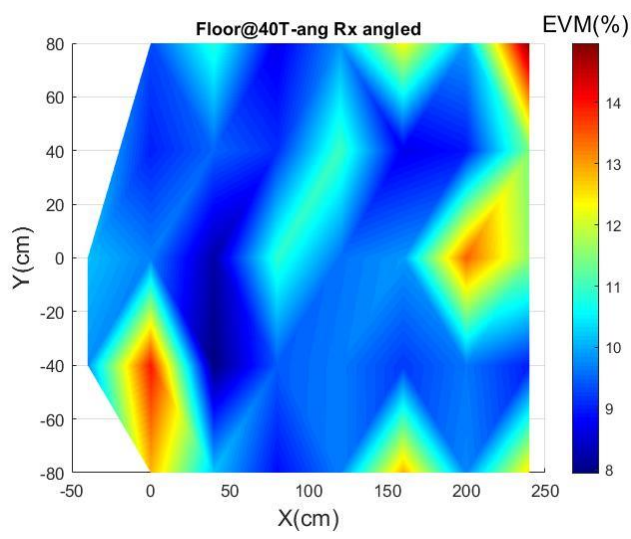

(b) With angling Rx toward Tx antenna

Figure 17: One mmWave TXs angled at 40o, receiver at $0 \mathrm{~m}$ above ground EVM Test

The results in Figure 17 show that the performance of the mmWave system does improve with Tx angling at 40 degrees.

\section{MMWAVE EMF EXPOSURE TEST RESULTS}

Due to the size of the simulation model and frequencies involved, WinProp was used for the main simulations and these results are shown below.

\section{A. Dominant electric field}

In ProMan, a combination of two result files is possible to get a result file, which contains the maximum, the minimum or the mean value of the two selected result files. To demonstrate direct comparisons between different transmitters and frequencies, combination of maximum value for each individual transmitter is done in this section (as described presently).

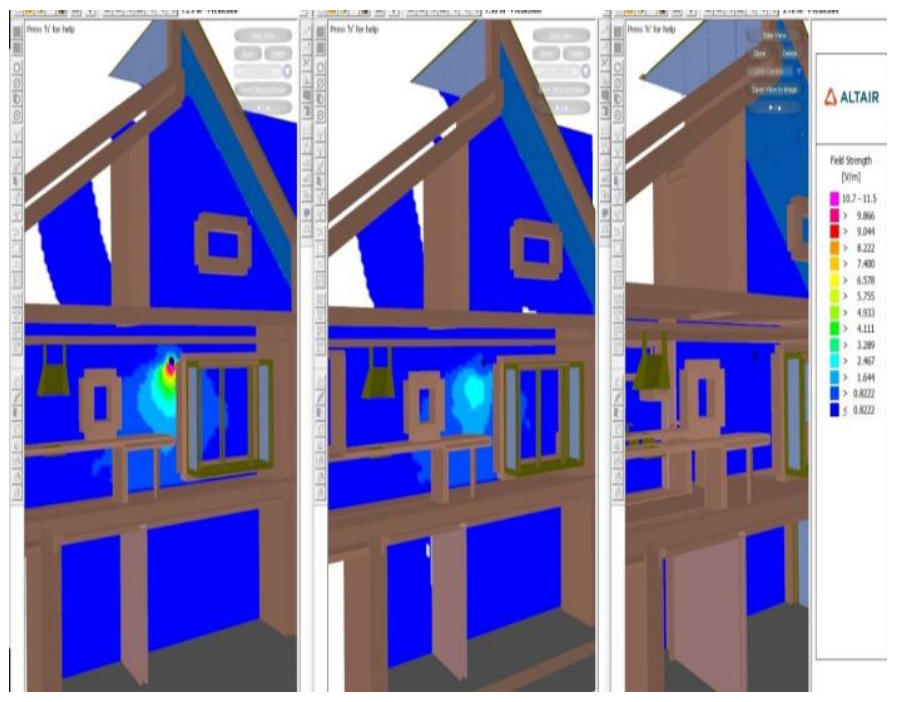

Figure 18: Single mmWave E-Field strength for all vertical prediction planes (most left: $1.29 \mathrm{~m}$; middle: $1.5 \mathrm{~m}$; most right: $3.10 \mathrm{~m}$ ). V/m linear scale

To set the scene and for better understanding of prediction planes see Figure 18, where the left-most image illustrates the vertical top-down distribution of energy of the lone mmWave 
This article has been accepted for publication in a future issue of this journal, but has not been fully edited. Content may change prior to final publication. Citation information: DOI10.1109/TBC.2021.3120918, IEEE Transactions on Broadcasting

transmitter modelled in the home. The absolute peak of the electric field strength is below $11 \mathrm{~V} / \mathrm{m}$, and this is located at the immediate excitation of the antenna, or even at the core of source placement. However, as the distance increases, the intensity of the electric field drops significantly and we can estimate that at the beginning of the far field region, values are in the range of below $2 \mathrm{~V} / \mathrm{m}$ (see vertical prediction plane in Figure 19).

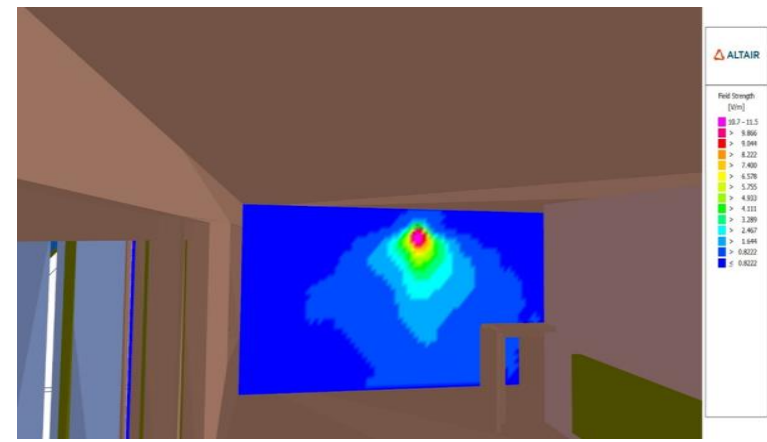

Figure 19: Single mmWave E-Field strength for the first vertical prediction plane. V/m linear scale

Figure 20 demonstrates another vertical prediction plane of electric field distribution per transmitter in V/m, whilst Figure 21 illustrates outcomes of power in logarithmic scale in $\mathrm{dBm}$.

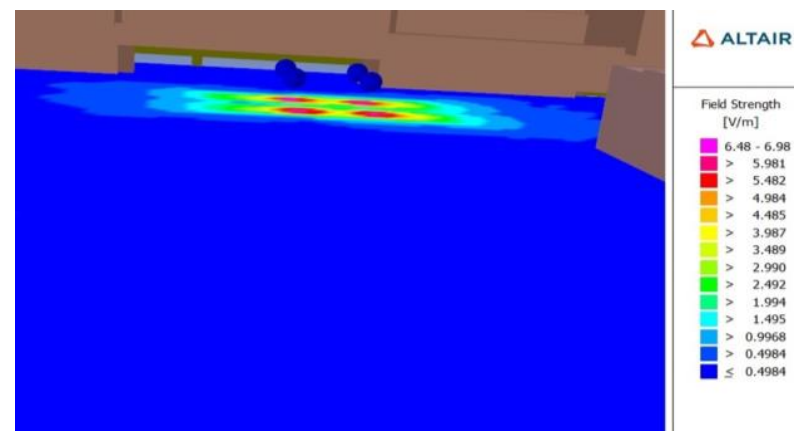

Figure 20: Indoors 3D view of four mmWave transmitters at height of $1.8 \mathrm{~m}$

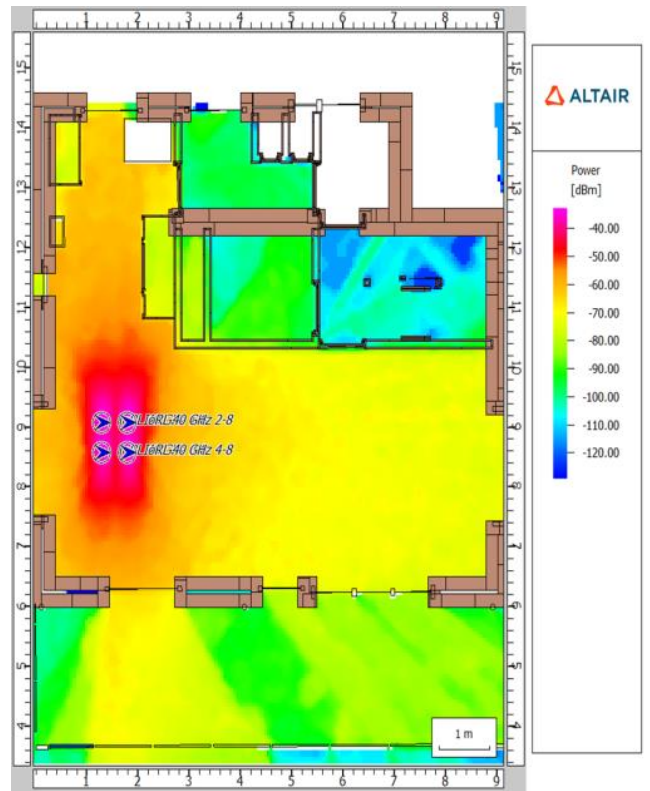

Figure 21: Four mmWave transmitters power level at height of $1.8 \mathrm{~m}$. Log scale

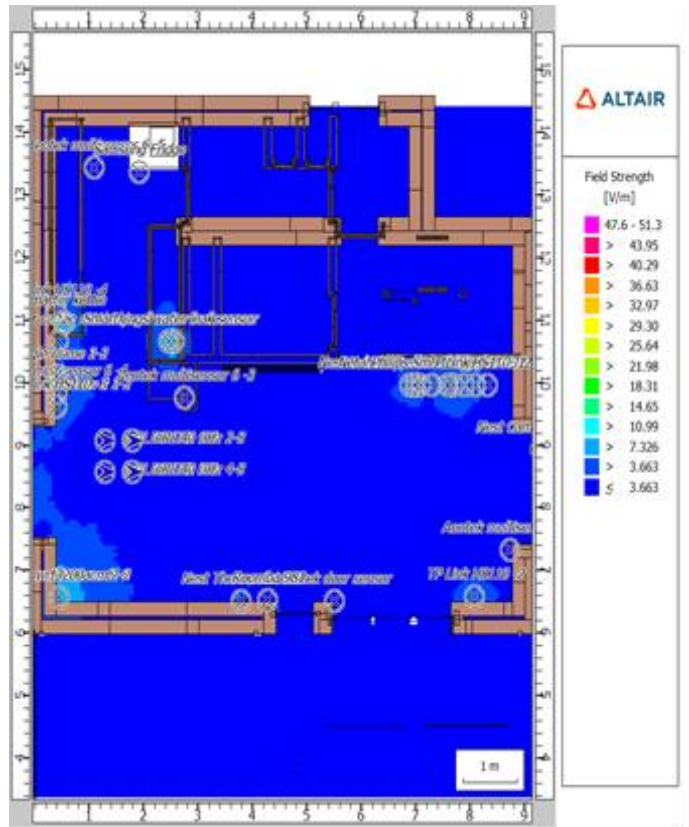

Figure 22: Simulation of E-Field strength for $4 \mathrm{mmWave}$ Tx and 40 additional Tx at height of $0.70 \mathrm{~m}$

\section{1) Total electric field}

To show the total field in relation to exposure limits, summation of exposure ratios is performed. It is important to determine whether, in situations of simultaneous exposure to fields of different frequencies, these exposures are additive in their effects.

For thermal considerations, relevant above $100 \mathrm{kHz}$ [8], the following two requirements should be applied to the field levels:

$$
\sum_{i=100 \mathrm{kHz}}^{1 \mathrm{MHz}}\left(\frac{E_{i}}{c}\right)^{2}+\sum_{i>1 \mathrm{MHz}}^{300 \mathrm{GHz}}\left(\frac{E_{i}}{E_{L, i}}\right)^{2} \leq 1
$$

and

$$
\sum_{i=100 \mathrm{kHz}}^{1 \mathrm{MHz}}\left(\frac{H_{i}}{d}\right)^{2}+\sum_{j>1 \mathrm{MHz}}^{300 \mathrm{GHz}}\left(\frac{H_{j}}{H_{L, j}}\right)^{2} \leq 1
$$

Where

$$
\begin{aligned}
& E_{i}=\text { the electric field strength at frequency } i ; \\
& E_{L, i}=\text { the electric field reference level } \\
& H_{j}=\text { the magnetic field strength at frequency } i ; \\
& H_{L, j}=\text { the magnetic field reference level } \\
& c=610 / f \mathrm{~V} / \mathrm{m}(f \text { in } M H z) \text { for occupational exposure and } \\
& 87 / f^{\wedge}(1 / 2) \mathrm{V} / \mathrm{m} \text { for general public exposure; and } \\
& d=1.6 / f \mathrm{f} / \mathrm{m}(\mathrm{f} \text { in } \mathrm{MHz}) \text { for occupational exposure and } 0.73 \\
& \qquad / f \text { for general public exposure }
\end{aligned}
$$

The total field strength was calculated for each of the device frequencies at the worst-case location just below the IoRL antennas and compared to the exposure limits and the calculated Exposure Ratios, TABLE 3. The total contribution to Exposure Ratio from all frequencies rounds up to 0.099 (ER $<1)$ so this is considerably below the ICNIRP limits. 
This article has been accepted for publication in a future issue of this journal, but has not been fully edited. Content may change prior to final publication. Citation information: DOI10.1109/TBC.2021.3120918, IEEE Transactions on Broadcasting

TABLE 3

Summation of Exposure Ratio contributors (in-phase constructive interference at a point - at the antenna)

\begin{tabular}{|l|l|l|l|l|l|}
\hline $\begin{array}{l}\text { Frequency } \\
\text { (MHz) }\end{array}$ & 868 & 1850 & 2400 & 5000 & 40000 \\
\hline Sum E-Field & 0.53 & 0.12 & $\begin{array}{l}15.84 \\
\mathrm{~V} / \mathrm{m}\end{array}$ & $\begin{array}{l}4.52 \\
\mathrm{~V} / \mathrm{m}\end{array}$ & $\begin{array}{l}9.791 \\
\mathrm{~V} / \mathrm{m}\end{array}$ \\
\hline E-Field limit & 40.51 & $\begin{array}{l}59.14 \\
\mathrm{~V} / \mathrm{m}\end{array}$ & $\begin{array}{l}61 \\
\mathrm{~V} / \mathrm{m}\end{array}$ & $\begin{array}{l}61 \\
\mathrm{~V} / \mathrm{m}\end{array}$ & $61 \mathrm{~V} / \mathrm{m}$ \\
\hline $\begin{array}{l}\text { Individual } \\
\text { contribution } \\
\text { to exposure } \\
\text { ratio }\end{array}$ & 0.000 & 0.00000 & 0.067 & 0.005 & 0.02576 \\
4712 & 4117 & 43 & 491 & \\
\hline
\end{tabular}

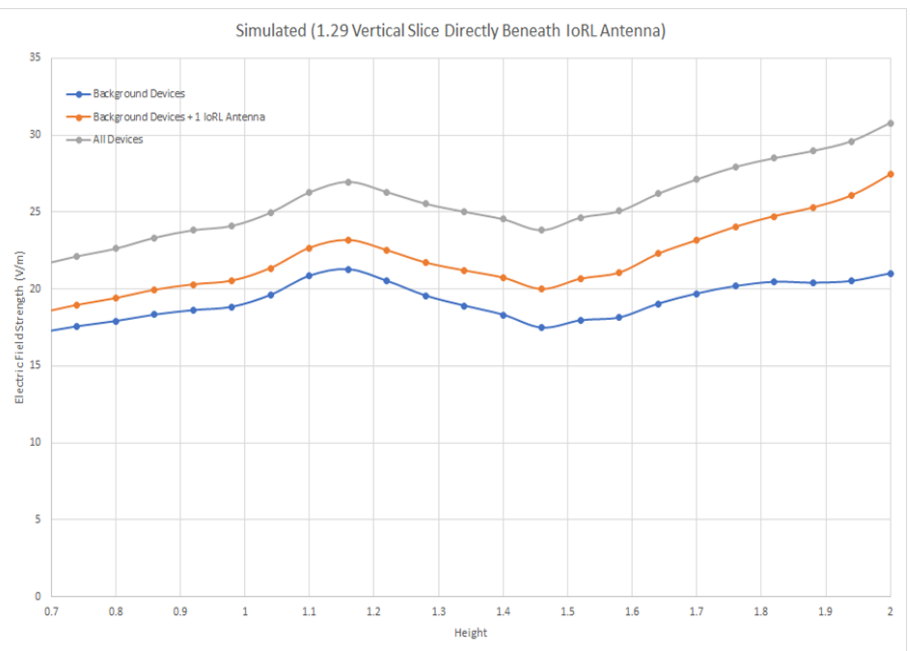

Figure 23: Summed broadband electric field strength at a point for all heights, in a vertical line, directly below mmWave antenna

Figure 23 shows the total field strength in the region directly below mmWave antenna, when all devices are summed assuming the worst case that they are all in-phase (constructive interference). It can be observed that there is a spike at 1.15 meters, where majority of IoT devices are vertically located although even higher field strength is observed, as the height approaches the mmWave radiation source height. Comparing the blue and orange lines in that graph, shows that the $40 \mathrm{GHz}$ contribution to the total electric field increases with elevation. It should be noted that this is a somewhat worst-case calculation and that in reality total field strengths are likely to be much lower, which was observed during the measurements detailed below.

\section{2) Measurements}

\section{a) Measurement Instrument selection}

The instrument used to perform the above measurements was a NARDA Broadband Field Meter NBM-520. The NARDA Broadband Field Meter NBM-520 is a popular instrument for measuring non-ionising radiation within the frequency range from $100 \mathrm{kHz}$ to $60 \mathrm{GHz}$ (depending on the probe used). Probes for various measurement applications are connected to the NBM-520 basic unit. Flat frequency response probes are also available, and shaped probes that evaluate the field according to a specific human safety standard. These probes are calibrated separately from the measuring instrument. Also, they include a non-volatile memory containing the probe parameters and calibration data. Therefore, they could be used with any instrument in the NBM-500 family without any lack in calibration accuracy.

The NBM-520 makes measurements for human safety purposes, mostly in workplace environments, where high electric or magnetic field strengths are expected. Also, it can be used to determine the electromagnetic compatibility (EMC) of devices and equipment. Examples:

- Measuring field strengths as part of general safety regulations

- Measuring the field strengths around transmitting and radar equipment to establish safety zones and for monitoring during operations

- Measuring the field strength emanating from mobile phone repeaters and satellite communications systems to ensure compliance with human safety limit values

- Measuring the field strength in the industrial workplace, such as tempering, RF heating, drying and plastics welding equipment.

- Field strength measurements in absorber chambers and TEM cells.

The measurement probe was selected to be EF4091.The probe contains three orthogonally arranged dipoles with detector diodes. The diode voltages each correspond to the RMS value of the spatial components.

The isotropic measurement result is obtained by addition within the probe. The probe detects electric fields from 40 $\mathrm{MHz}$ up to $40 \mathrm{GHz}$. This frequency range covers almost the entire range of high frequency communications, right up to mobile radio and satellite links. The linearity and sensitivity of the probe ensure its suitability for checking human safety limit values in the occupational and general public environments.

\section{b) Measurements - procedures and methodologies}

The aluminium frame on which the RRLH controller and RRLHs are fitted is shown in Figure 24 in order for the mmWave antenna location to coincide with the mmWave antenna location used in the EM radio simulations, as shown in Figure 21 and Figure 22.

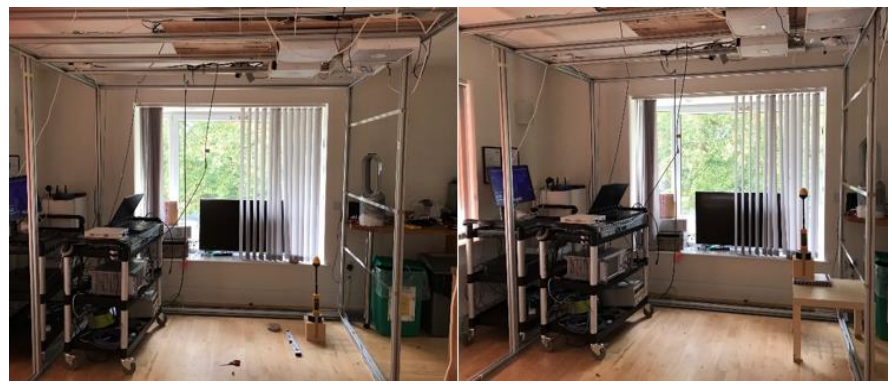

(a) At $0.7 \mathrm{~m}$ height

(b) At $1.2 \mathrm{~m}$ height

Figure 24: Location of RRLH Controller and RRLHs for EM Radiation Measurements 
The location of the source and RF propagation path during measurements were considered to minimise the influence of the body on the result. A check was made, as per the manufacturer's specifications for the minimum distance between the measurement probe tip and the body of the "operator", as well as to any reflecting object. Non-conductive materials were used, to secure and position measuring device in place. For handheld measurements, the uncertainty due to the scattering of the RF field by the surveyor's body was minimised by:

- holding the probe or antenna away from the surveyor's body (a separation of at least $50 \mathrm{~cm}$ should be maintained between the measurement antenna or isotropic probe and the surveyor's body);

- pointing the probe towards the source;

- ensuring that the surveyor's body is not along the direct line of propagation between the source and the measurement probe (either in front of or behind).

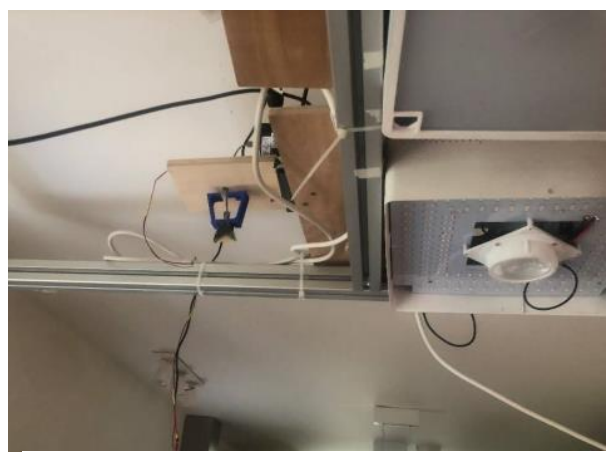

Figure 25: Location of mmWave Antenna

The Intelligent Home IP Gateway, Layer 2 Processor and DRAN was located on one trolley as shown in Figure 26a, whilst the Viavi User Test Terminal was located on a second trolley, as shown in Figure 26b. The EM radiation level measurement device, shown in Figure 27, can operate independently to the Test End User Terminal.

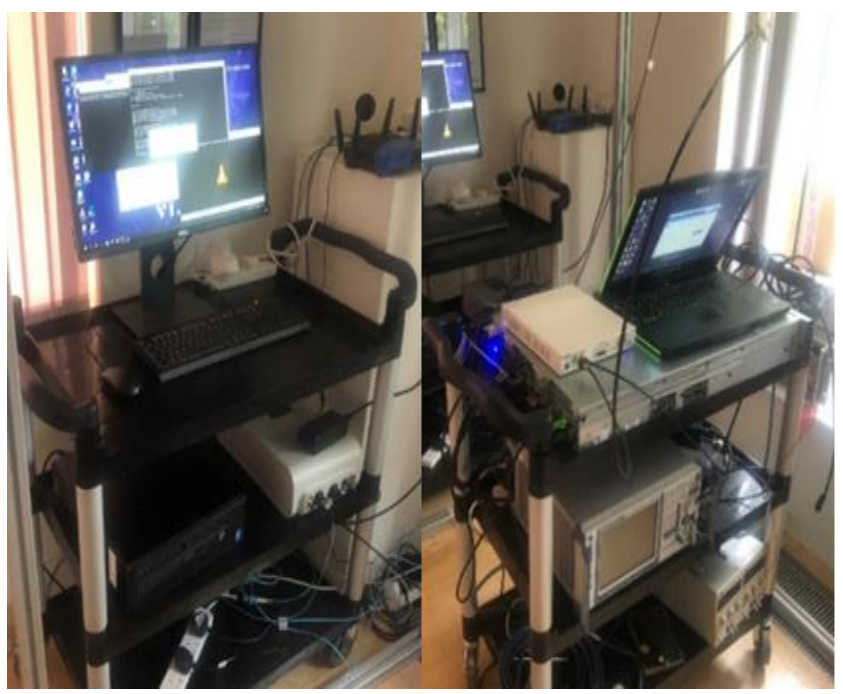

(a) Intelligent Home IP

Gateway, Layer 2 Processor \& DRAN

(b) Viavi Test End User Terminal
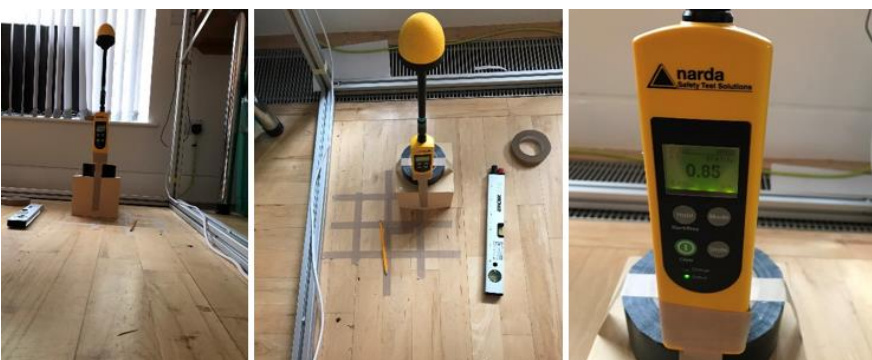

Figure 27: EM Radiation Level Measurement device

The system was set to broadcast at full power. Measurements were performed at three heights: 0.7, 1.2 and 1.8 meters above floor level. Theoretically, at $1.8 \mathrm{~m}$, the colleagues collecting data are in the Near Field region of the antenna.

Two measurement procedures were performed: with the IoRL system ON and OFF. Additionally, space averaging, peak and time averaging measurements were done.

Space averaging Discreet spatial measurements were performed for each horizontal prediction plane, averaging over a $3 \times 3$ grid ( 9 points, the point in the middle of grid, marked as $\mathrm{X} 5$, is located directly below mmWave transmitter - see Figure 28 below), with $10 \mathrm{~cm}$ distance between each measurement point.

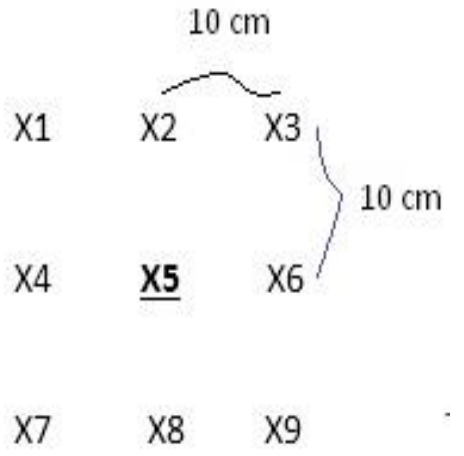

Figure 28: Diagram of measurement grid

c) Results

(1) mmWave System turned ON

1. Time averaging peak measurement over 6 minutes, directly below transmitting antenna (probe physically in contact with antenna)

Result is listed below:

$$
\text { a. } 5.74 \mathrm{~V} / \mathrm{m}
$$

2. Spatial averaging measurement over 9 points consisting of square $3 \times 3$ grid, with distance of $10 \mathrm{~cm}$ between points and middle of grid directly under mmWave antenna, at each height.

Results are listed in TABLE 4: 
This article has been accepted for publication in a future issue of this journal, but has not been fully edited. Content may change prior to final publication. Citation information: DOI10.1109/TBC.2021.3120918, IEEE Transactions on Broadcasting

TABLE 4

Measured electric field strength $(\mathrm{V} / \mathrm{m})$, spaced averaged, with mmWave transmitter turned on

\begin{tabular}{|l|l|l|l|}
\hline $\begin{array}{l}\text { Meas. Point } \\
\text { Height }\end{array}$ & $\begin{array}{l}\text { Height 1 } \\
\mathbf{=}=\mathbf{0 . 7} \mathbf{~ m}\end{array}$ & $\begin{array}{l}\text { Height 2 } \\
\mathbf{= = 1 . 2 ~} \mathbf{~ m}\end{array}$ & $\begin{array}{l}\text { Height 3 } \\
\mathbf{= = 1 . 8} \mathbf{~ m}\end{array}$ \\
\hline X1 & 1.02 & 1.24 & 1.61 \\
\hline X2 Averages & 0.92 & 1.15 & 1.62 \\
\hline X3 Averages & 0.85 & 1.05 & 1.69 \\
\hline X4 Averages & 0.87 & 1.05 & 1.75 \\
\hline X5 Averages & 0.82 & 1.06 & 1.80 \\
\hline X6 Averages & 0.82 & 1.08 & 1.79 \\
\hline X7 Averages & 0.90 & 1.08 & 1.70 \\
\hline X8 Averages & 0.88 & 1.05 & 1.68 \\
\hline X9 Final Average & $\mathbf{0 . 8 9}$ & $\mathbf{1 . 0 6}$ & $\mathbf{1 . 6 5}$ \\
\hline
\end{tabular}

* Measurements were taken in the following sequence: $\mathrm{X} 1$, X2, X3, X6, X5, X4, X7, X8, X9.

3. Absolute peak measurement directly below transmitting antenna (probe physically in contact with antenna)

$$
\text { a. } 6.44 \mathrm{~V} / \mathrm{m}
$$

\section{(2) mmWave System turned OFF}

1. Time averaging peak measurement over 6 minutes, directly below transmitting antenna (probe physically in contact with antenna)

Result is listed below:

$$
\text { a. } 0.69 \mathrm{~V} / \mathrm{m}
$$

2. Spatial averaging measurement over 9 points consisting of square $3 \times 3$ grid, with distance of $10 \mathrm{~cm}$ between points and middle of grid directly under mmWave antenna, at each height.

Results are listed in TABLE 5:

TABLE 5

Measured electric field strength $(\mathrm{V} / \mathrm{m})$, spaced averaged, with mmWave transmitter turned off

\begin{tabular}{|l|l|l|l|}
\hline $\begin{array}{l}\text { Meas. Point } \\
\text { Height }\end{array}$ & $\begin{array}{l}\text { Height 1 } \\
\mathbf{=}=\mathbf{0 . 7} \mathbf{~ m}\end{array}$ & $\begin{array}{l}\text { Height 2 } \\
\mathbf{=}=\mathbf{1 . 2} \mathbf{~ m}\end{array}$ & $\begin{array}{l}\text { Height 3 } \\
\mathbf{= 1 . 8} \mathbf{~ m}\end{array}$ \\
\hline X1 & 0.63 & 0.82 & 1.22 \\
\hline X2 Averages & 0.47 & 0.74 & 1.29 \\
\hline X3 Averages & 0.54 & 0.66 & 1.32 \\
\hline X4 Averages & 0.60 & 0.60 & 1.25 \\
\hline X5 Averages & 0.56 & 0.60 & 1.32 \\
\hline X6 Averages & 0.54 & 0.63 & 1.34 \\
\hline X7 Averages & 0.58 & 0.64 & 1.19 \\
\hline X8 Averages & 0.58 & 0.67 & 1.18 \\
\hline X9 Final Average & $\mathbf{0 . 5 7}$ & $\mathbf{0 . 6 7}$ & $\mathbf{1 . 2 1}$ \\
\hline
\end{tabular}

* Measurements were taken in the following sequence: $\mathrm{X} 1$, X2, X3, X6, X5, X4, X7, X8, X9.

\section{ANALYSIS OF RESULTS}

\section{A. VLC coverage}

Results from VLC measurements shows that the coverage has a diameter of about $0.6 \mathrm{~m}$ and maximum propagation distance of $2 \mathrm{~m}$. This limited coverage was attributed to the physical construction of the PD sensor housing. The variation in performance between communication LEDs A, B, C and D was attributed to the variability in transmitted light intensities between them.

When angling the PD receiver towards the transmit LED, this improves the quality of the received signal so that the coverage has a diameter of about $1 \mathrm{~m}$ and maximum propagation distance of $2 \mathrm{~m}$, as shown in Figure 8. Again, the variation in performance between communication LEDs A, B, $\mathrm{C}$ and $\mathrm{D}$ was attributed to the variability in transmitted light intensities between them.

\section{B. VLC location}

Received signal strength results have also been used to locate positions with a minimum location error of $3.5 \mathrm{~cm}$ and $80 \%$ of all location measurement errors of less than $10 \mathrm{~cm}$.

\section{C. mmWave coverage}

Results from mmWave 64 QAM transmissions have shown that for a single polarisation transmit antenna pointing vertically down, there was a reasonably consistent coverage area of about two meters diameter at $0.7 \mathrm{~m}$ above ground $(1.3 \mathrm{~m}$ from transmit antenna) with and without angling the receive antenna towards the transmit antenna, as shown in Figure 12. There was a slight improvement of results when angling the receive antenna towards the transmit antenna. The asymmetry of the coverage performance was attributed to a glass door on one side of the coverage area, which produced mmWave reflections that impaired the performance of the receiver. An improved design of the mmWave, which more effectively processes multipath propagations, would provide more symmetric coverage performance results.

Results from mmWave 64 QAM transmissions have shown that for a single polarisation transmit antenna pointing vertically down, there was a patchy coverage area of about two meters diameter at $0.0 \mathrm{~m}$ above ground $(2.0 \mathrm{~m}$ from transmit antenna) with and without angling the receive antenna towards the transmit antenna, as shown in Figure 13. There was no noticeable improvement of results when angling the receive antenna towards the transmit antenna. Again, the asymmetry of the coverage performance was attributed to a glass door on one side of the coverage area, which produced mmWave reflections that impaired the performance of the receiver.

When the transmit antenna was rotated towards the receive antenna at 30 degrees from the vertical, a patchy coverage area was measured, which has a width of $2.0 \mathrm{~m}$ and length of at least $1.6 \mathrm{~m}$ at $0.0 \mathrm{~m}$ above ground level when the receive antenna is rotated towards the transmit antenna, as shown in Figure b. When the receive antenna was not angled towards the transmit antenna, the coverage area has been reduced to a width and length of about $1 \mathrm{~m}$, as shown in Figure a. When the same experiment was repeated five days later on the 22nd of 
September, similar performance coverage area results were obtained but with different patchy patterns, as shown in Figure $\mathrm{a}$ and $15 \mathrm{~b}$, the difference of which were not able to be explained.

When the transmit antenna was rotated towards the receive antenna at 30 degrees from the vertical, a uniform coverage area was measured, which has a width of $2.0 \mathrm{~m}$ and length of more than $2.0 \mathrm{~m}(6 \mathrm{~m}$ as measured in laboratory) at $0.7 \mathrm{~m}$ above ground level when the receive antenna is rotated towards the transmit antenna, as shown in Figub. When the receive antenna was not angled towards the transmit antenna, the coverage area has the same width but a reduced length of about $1 \mathrm{~m}$, as shown in Figua.

When the transmit antenna was rotated towards the receive antenna at 30 degrees from the vertical, a patchy coverage area was measured, which has a width of $2.0 \mathrm{~m}$ and length of $2.0 \mathrm{~m}$ at $0.0 \mathrm{~m}$ above ground level when the receive antenna is rotated towards the transmit antenna, as shown in Figure 17b. When the receive antenna was not angled towards the transmit antenna, the coverage area has a reduced width of $1.6 \mathrm{~m}$ and reduced length of about 1m, as shown in Figure 17a.

\section{D. mmWave EM Exposure}

The measurements and simulations are compared in Figure 29 and Figure 30. The orange and blue line in Figure 29 denote calculations that are based on the simulated data, and were performed assuming perfect in-phase constructive interference, as this represent the worst-case scenario possible, although it is recognised that this is not realistic in practice and the measurements confirm this.

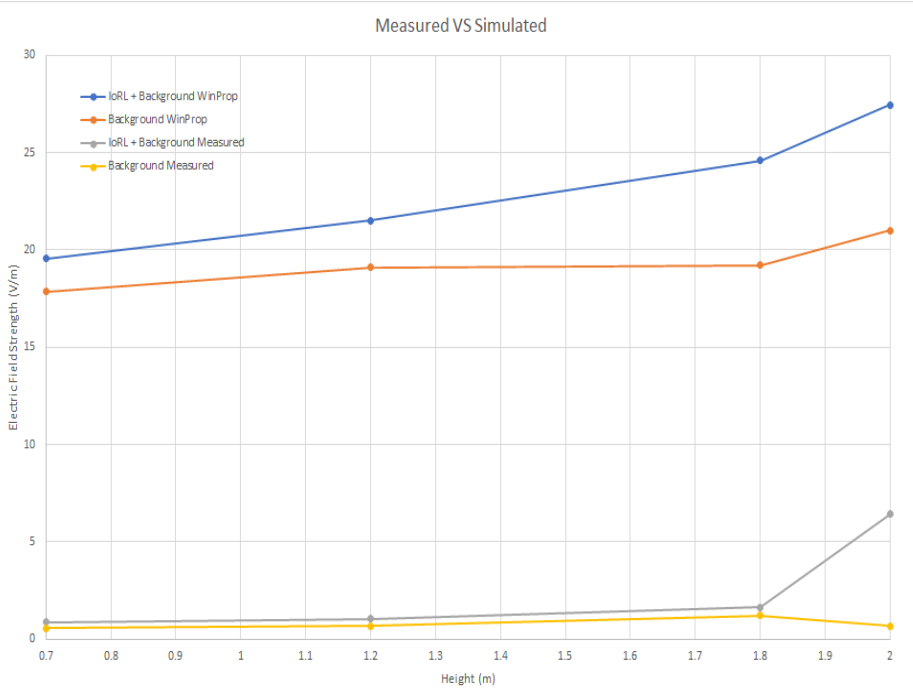

Figure 29:Comparisons between measured and simulated results (Dominant electric field)

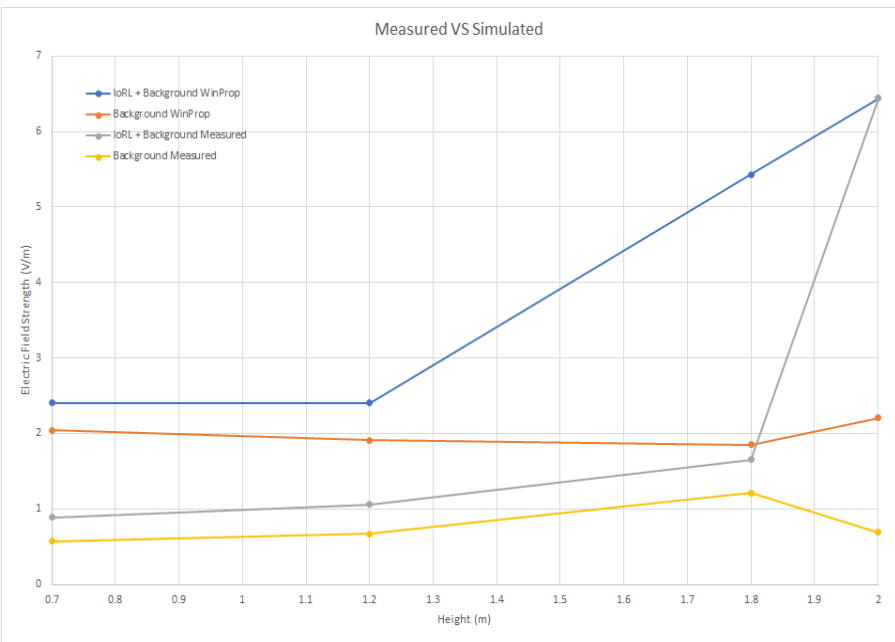

Figure 30: Comparisons between measured and simulated results (summation of electric field strengths, assuming in-phase constructive interference)

In Figure 30, the measurements are compared to the simulated dominant field. This shows a much better comparison. At a height of $2 \mathrm{~m}$, where the mmWave transmitter is located, there is a very good match, with both the predicted and measured field strengths being about $6.4 \mathrm{~V} / \mathrm{m}$, for the mmWave and background radiation. The main difference is between the measurement and simulation of the background field strengths, with the measurements average about $1 \mathrm{~V} / \mathrm{m}$ and the simulations $2 \mathrm{~V} / \mathrm{m}$ over the range of heights.

\section{CONCLUSIONS}

The level of performance that was measured for the $5 \mathrm{G}$ VLC communications system is more suited to Personal Communication applications such as that required for transmission to passengers on seating within aircraft and trains or for controlling home devices such as washing machines and TVs from smart phones. Performance of the VLC communications system would have to increase propagation distance by a factor of 3 to $6 \mathrm{~m}$ and increase the coverage area by a factor of six to 6 meters for it to be a general-purpose communications competitor to the mmWave communication system in indoor environments. Furthermore, enhancements to the PD receiver would also be required so that it has the multidirectional photo sensing properties of a fly's eye, which could possibly be achieved using a Fresnel lens at the receiver.

The level of performance that was measured for the $5 \mathrm{G}$ mmWave communications system has shown the viability of a $5 \mathrm{G}$ networked home since just four mmWave radio heads would be required to provide sufficient coverage for a family sized sitting room, whilst also providing sufficient numbers of mmWave radio access point to be able to measure location. The best position of these radio heads is at the four corners of the room pointing at 30 degrees from the vertical towards the centre of the room. The transmit antenna would be required to be enhanced so that it is circular or polarised or at the least provide both vertical and horizontal polarised antennas transmitters. Further experiments need to be performed from four transmit antennas to show that this man-made multipath 
environment does away with the requirement of angling the receive antenna towards any one transmits antenna to obtain improved performance, which would be physically impossible to achieve when simultaneously transmitting the same radio signal from four different transmit antennas at the same time. Implementation of the mmWave Time Division Multiplexing return channel means that the next phase of the measurement campaign will consist of measuring the accuracy of Time Difference of Arrival location.

The ICNIRP exposure ratio calculated from the total field strength contributions from the simulated results estimated that the exposure ratio was just less than 0.1 (see Table 3), with any value below one being compliant with the ICNIRP limits, and this was therefore considerably below the ICNIRP limits. The measurement field strengths were slightly lower than the simulations and reasonably similar to the simulated results.

This work gives an indication of the estimated levels of risk associated with the scenarios modelled. However, this work should not be taken as any kind of approval for such products to be placed for sale on the market. Any manufacturers placing such products on the market should go through the necessary product approval processes to meet the necessary regulations and standards, including any national standards and guidance, and perform their own assessments of their specific system specifications.

International Committee on Non-Ionizing Radiation Protection (ICNIRP) guidelines are used worldwide, either directly or as the basis for national regulations. These ICNIRP levels have been used in this report as a reference level. It is recognised that there are some variations in some national regulations, and these should be considered for any product manufacturers looking to take products to market.

The emissions from other wireless devices in addition to IoRL were included in the calculations to give estimates of other background field strength levels. However, this report is only assessing the potential impact of the IoRL devices with respect to human exposure limits, not any other wireless devices.

Some calculations use a number of worst-case assumptions, such as the contributions from all devices being in-phase and all with $100 \%$ activity. These results should not be taken as a representation of actual field strengths that would be experienced in practice. Measurements were made of actual devices which considered how the devices operate in practice and demonstrated lower levels of field strength as expected.

\section{ACKNOWLEDGMENT}

The authors gratefully acknowledge the European Commission for its financial support on the Horizon 2020 Internet of Radio-Light (IoRL) project No: 761992.

\section{REFERENCES}

[1] Yue Zhang, Hequn Zhang, John Cosmas, Nawar Jawad, Kareem Ali, Ben Meunier, Adam Kapovits, Li-Ke Huang, Wei Li, Lina Shi, Xun Zhang, Jintao Wang, Israel Koffman, Muller Robert, and Charilaos C. Zarakovitis "Internet of Radio and Light: 5G Building Network Radio and Edge Architecture" ITU Journal of Intelligent and Converged Networks, Volume 1, the 01st of March 2020, DOI:10.26599/ICN.2020.9070002, Online: 2020-04-24
[2] Lina Shi, Dayu Shi, Xun Zhang, Benjamin Meunier, Hequn Zhang, Zhan Wang, Andrei Vladimirescu, Wei Li, Yue Zhang, John Cosmas, Kareem Ali, Nawar Jawad, Rudolf Zetik, Eric Legale, Matteo Satta, Jintao Wang, and Jian Song "5G Internet of Radio Light Positioning System for Indoor Broadcasting Service" IEEE TRANSACTIONS ON BROADCASTING, VOL. 66, NO. 2, JUNE 2020

[3] ITU-R BS.1698, Evaluating fields from terrestrial broadcasting transmitting systems operating in any frequency band for assessing exposure to non-ionising radiation.

[4] ITU-T K.61, guidance on measurement and numerical prediction of electromagnetic fields for compliance with human exposure limits for telecommunication installations, Geneva, Switzerland, 2018.

[5] Kathy L. Ryan, John A D'Andrea, James R. Jauchem, Patrick A Mason, "Radio frequency radiation of millimeter wave length: potential occupational safety issues relating to surface heating," 2000.

[6] "IORL 5GPPP," [Online]. Available: https://iorl.5g-ppp.eu/. [Accessed $28012019]$.

[7] IEEE Std 1720 Antenna Measurements.

[8] ICNIRP, ICNIRP Guidelines for limiting exposure to time-varying electric, magnetic and electromagnetic fields (UP TO $300 \mathrm{GHZ}$ ), 1998.

[9] ITU-T K.52, guidance on complying with limits for human exposure to electromagnetic fields, 2018.

[10] IEC 62630, guidance for evaluating exposure from multiple electromagnetic sources - Technical Report.

[11] ITU-T K.91, guidance for assessment, evaluation and monitoring of human exposure to radio frequency electromagnetic fields, Geneva, Switzerland, 2018.

[12] EBU - European Broadcasting Union, EBU [November 2001] BPN 023: Radio frequency radiation: Exposure limits and their implication for broadcaster., 2001.

[13] EN 62311:2008, Assessment of electronic and electrical equipment related to human exposure restrictions for electromagnetic fields $(0 \mathrm{~Hz}-300$ GHz), 2008.

[14] IEC 62232, EN 62232, Determination of RF field strength, power density and SAR in the vicinity of radiocommunication base stations for the purpose of evaluating human exposure, 2017.

[15] ITU-T K.91, guidance for assessment, evaluation and monitoring of human exposure to radio frequency electromagnetic fields, Geneva, Switzerland, 2018.

[16] John Cosmas; Yue Zhang; Ben Meunier; Kareem Ali "IoRL Measurement Campaign Data" DOI: 10.5281/zenodo.4580334, https://doi.org/10.5281/zenodo.4580334

[17] Atanas Savov; James Gbadamosi "Materials Property Database for EM Exposure Simulation" DOI 10.5281/zenodo.4580351, https://doi.org/10.5281/zenodo.4580351 